\title{
Comprometimento cognitivo e demência na neurocisticercose ativa: um estudo transversal controlado
}

Tese apresentada à Faculdade de Medicina da Universidade de São Paulo para obtenção do título de Doutor em Ciências

Programa de: Neurologia

Orientador: Prof. Dr. Paulo Caramelli 
Dados Internacionais de Catalogação na Publicação (CIP)

Preparada pela Biblioteca da

Faculdade de Medicina da Universidade de São Paulo

Creprodução autorizada pelo autor

Andrade, Daniel Campi Araujo de

Comprometimento cognitivo e demência na neurocisticercose ativa : um estudo transversal controlado / Daniel Campi Araujo de Andrade. -- São Paulo, 2010.

Tese(doutorado)--Faculdade de Medicina da Universidade de São Paulo.

Programa de Neurologia.

Orientador: Paulo Caramelli.

Descritores: 1.Neurocisticercose 2.Cognição 3.Manifestações neurocomportamentais 4.Demência 5.Epilpesia

USP/FM/DBD-248/10 


\section{Dedicatória}

Esta tese é dedicada à minha família.

$\grave{A}$ minha esposa pelo sinergismo em tudo que faço penso e sinto, aos meus pais e irmã pela base e o caminho, e meu filho pelo objetivo. 


\section{Agradecimentos}

Aos pacientes, que permitiram que o seu sofrimento fosse quantificado, transformado em estatística e retransmitido a outros. Na esperança de nos tornar menos ignorantes nesta matéria da ciência médica. Na esperança de que outros, que eles nunca conhecerão, sejam acolhidos e tratados de forma mais precisa do que eles o foram.

Cleonísio Leite Rodriques. Irmão neurologista, amigo e parceiro em todas as fases e empreitadas deste projeto. Este trabalho é tão dele quanto meu.

Paulo Caramelli. Idealizador do projeto, foi neste período todo um Orientador. Absolutamente todas as propostas que propôs foram frutíferas. Quanto maior a distância física entre ele e seu orientando, sua disponibilidade e paciência aumentavam de forma exponencial. Teleconferências transatlânticas de madrugada são só um exemplo. Sua seriedade, pontualidades e bom senso agudo só me inspiram e norteiam meus parâmetros sobre aonde devo chegar.

Luis dos Ramos Machado. Meu co-orientador que moldou todo o trabalho para que respondesse às perguntas mais pertinentes na literatura. Seu senso critico de clínico e pesquisador enriqueceu a metodologia e a fortaleceu a tal ponto que conseguiu enfrentar com sucesso o mar dos mais tenebrosos revisores internacionais.

Ronaldo Abram: amigo e incentivador. Deu novo fôlego a esta nova fase de pesquisas em neurocisticercose. Foi incansavelmente disponível para nos auxiliar, nos encaminhar doentes e avaliá-los do ponto de vista iconográfico e clínico.

Antônio Livramento: enriqueceu o trabalho com seu conhecimento clínico e abriu portas para todas as nossas propostas. Grande incentivador de minha geração de neurologistas.

Cláudia Leite: Moldou a literatura internacional em imagem e neurocisticercose, junto com Leandro Lucato e seus assistentes, participaram de forma ativa com nosso estudo e o enriqueceram.

Luis Henrique Castro: um dos melhores neurologistas que conheci até hoje. Grande capacidade sintética e objetividade. A ele agradeço não somente por ter aberto as portas de seu ambulatório de epilepsia e ter dado sugestões riquíssimas ao estudo, mas também por tudo que me ensinou durante os anos de residência. Altruísta, repassa sem rodeios pérolas da neurologia que tenho certeza lhe custaram anos de estudo árduo. Espero que as novas gerações de neurologistas possam tê-lo como mestre, assim como nós temos. 
Professor Manoel Jacobsen Teixeira: Professor por titulo e comportamento. Empreendedor, planejador, incentivador. Defensor e exemplo da medicina voltada ao doente, ao exame físico, à percepção e observação detalhada. Antes de neurocirurgião, neurologista. Antes de neurologista, medico. Antes de medico, humano. A ele só agradeço. Por todas as oportunidades, por todos os incentivos. Ao apoio nos momentos conturbados. À cobrança para se melhorar o que já é bom. Ao pioneirismo. E ao total desprendimento ao conhecimento médico, que distribui abertamente a todos que cruzam seu caminho. Obrigado pelo apoio a este trabalho e por ser meu orientador na vida médica e pessoal.

Professor Milberto Scaff. Permitiu a realização deste trabalho em seu departamento, que o financiou direta e indiretamente. Agradeço o apoio que sempre ofereceu aos meus esforços no Departamento.

Professor Ricardo Nitrini. Incentivador deste trabalho desde o início, agradeço pelo encorajamento e comentários que realizou durante a qualificação, que só enriqueceram este trabalho

Prof Paulo E. Marchiori. Incentivou-me na realização deste estudo, alem de permitir-me utilizar minhas matérias optativas do quarto ano de residência em neurologia para desenvolver parte da coleta de dados do estudo.

Sônia Brucki. Agradeço a leitura atenta e critica da versão da qualificação, bem como os cometários que permitiram um aprimoramento do texto e da discussão.

Caudia Porto. Teve participação ativa em nosso treinamento práticoteórico em testes neuropsicológicos.

Às Sras. Márcia Benetti, Valdenira dos Santos Silva, Vanessa Ramos, Sipriana S.A. Santos, Sueli Martins, Reiko Uchizono Simomura, Vera Lúcia Pereira, Elisabete da Silva B. Malagodi, Vera Maria de Paula dos Santos Daniela Cristina N. $\operatorname{dos}$ A. Rosa.

Às Enfermeiras Maria de Lurdes P. Santos, Sandra Celidonia da Silva.

Ao Conselho Nacional de Pesquisa e Desenvolvimento Tecnológico (CNPq) pela bolsa de doutorado que recebi.

\section{Epígrafe}


Fort hereusement, chaque réussite est l'échec d'autre chose. Jacques Prévert (1900-1977) 


\section{Normas Adotadas}

Esta tese está de acordo com as seguintes normas, em vigor no momento desta publicação:

Referências: adaptado de International Committee of Medical Journals Editors (Vancouver)

Universidade de São Paulo. Faculdade de Medicina. Serviço de Biblioteca e Documentação. Guia de apresentação de dissertações, teses e monografias. Elaborado por Anneliese Carneiro da Cunha, Maria Julia de A. L. Freddi, Maria F. Crestana, Marinalva de Souza Aragão, Sueli Campos Cardoso, Valéria Vilhena. 2a ed. São Paulo: Serviço de Biblioteca e Documentação; 2005.

Abreviaturas dos títulos dos periódicos de acordo com List of Journals Indexed in Index Medicus. 


\section{Abreviações}

Bateria Breve de Rastreio Cognitivo (BBRC)

Comprometimento Cognitivo Sem Demência (CCSD)

Controles Saudáveis (CS)

"Diagnostic and Statistical Manual of Mental Disorders 4th edition" (DSMIV)

Drogas antiepilépticas (DAE)

Epilepsia Criptogênica (EC)

Extensão de dígitos em ordem direta (EDD)

Extensão de dígitos em ordem inversa (EDI)

Desenho do Relógio (DR)

Líquido cefaloraquidiano (LCR)

Memória verbal (MV)

Mini-Exame do Estado Mental (MEEM)

Neurocisticercose (NC)

Praxia Construtiva (PC)

Questionário de Atividades Funcionais de Pfeffer (PFAQ)

Ressonância Nuclear Magnética de crânio (RM)

Sistema Nervoso Central (SNC)

Teste de Interferência de Cores de Stroop (TICS)

Teste de Fluência Verbal Semântica (FVS)

Teste de nomeação (TN)

Teste de Trilhas A (TTA)

Teste de Trilhas B (TTB)

Tomografia Computadorizada de Crânio (TC) 
N. USP 2358420 EMAIL - ciampi@usp.br

Resumo: Comprometimento cognitivo e demência na neurocisticercose ativa: um estudo transversal controlado

Introdução: Neurocisticercose (NC) é a doença parasitária do SNC mais frequente no mundo. Afeta mais de 50 milhões de pessoas. No entanto, algumas de suas manifestações clínicas, como comprometimento cognitivo e demência, ainda permanecem caracterizadas de modo incompleto, sem que haja estudos controlados disponíveis na literatura até o momento.

Objetivos: Investigar a frequência e o perfil clínico do comprometimento cognitivo associado à NC ativa, comparando o desempenho em testes de avaliação cognitiva de pacientes com a doença ao de controles saudáveis (CS) e de pacientes com epilepsia criptogênica (EC).

Métodos: Quarenta pacientes (idade média $=39,25 \pm 10,50$ anos), com diagnóstico de NC ativa segundo critérios absolutos à ressonância magnética (RM) de crânio e sem tratamento antiparasitário prévio foram submetidos à avaliação cognitiva e funcional extensas, sendo comparados a 49 CS e 28 pacientes com EC emparelhados por idade, nível educacional e frequência de crises epilépticas (grupo EC).

Resultados: Pacientes com NC apresentaram comprometimento significativo em relação ao grupo CS nos testes que avaliam funções executivas, memória verbal e não verbal, praxia construtiva e fluência verbal $(p<0,05)$. Demência foi diagnosticada em $12,5 \%$ dos pacientes com NC de acordo com os critérios do DSM-IV. Os doentes do grupo NC apresentaram desempenho significativamente inferior em testes de memória operacional, memória episódica verbal, funções executivas, nomeação, praxia construtiva e orientação visual-espacial, quando comparados àqueles do grupo EC. Não se 
encontrou correlação entre as alterações nos testes cognitivos nos pacientes com NC e os achados à RM (carga de doença, tipo e localização das lesões).

Conclusões: Comprometimento cognitivo foi muito frequente na amostra de pacientes com NC avaliada, sendo que demência foi identificada em uma proporção significativa dos doentes. Estes dados aumentam o nosso conhecimento sobre a apresentação clínica da NC e sobre seu potencial impacto na saúde pública.

Palavras Chave: neurocisticercose, cognição, manifestações neurocomportamentais, demência, epilepsia. 
ABSTRACT: Cognitive impairment and dementia in neurocysticorsis a crosssectional controlled study

Introduction: Neurocysticercosis (NCYST) is the most frequent CNS parasitic disease worldwide, affecting more than 50 million people. However, some of its clinical findings, such as cognitive impairment and dementia, remain poorly characterized, with no controlled studies conducted so far. We investigated the frequency and the clinical profile of cognitive impairment and dementia in a sample of NCYST patients in comparison to cognitively healthy controls (HC) and to cryptogenic epilepsy (CE) patients.

Methods: Forty treatment-naïve NCYST patients aged $39.25 \pm 10.50$ years and fulfilling absolute criteria for definitive active NCYST on magnetic resonance imaging ( $\mathrm{MRI})$, underwent a comprehensive cognitive and functional evaluation and were compared to $49 \mathrm{HC}$ and $28 \mathrm{CE}$ patients of similar age, educational level, and seizure frequency.

Results: NCYST patients displayed significant impairment in executive functions, verbal and non-verbal memory, constructive praxis, and verbal fluency when compared to $\mathrm{HC}(\mathrm{p}<0.05)$. Dementia was diagnosed in $12.5 \%$ of NCYST patients according to the DSM-IV criteria. When compared to CE patients, NCYST patients presented altered working and episodic verbal memory, executive functions, naming, verbal fluency, constructive praxis, and visual-spatial orientation. No correlation emerged between cognitive scores and number, localization or type of NCYST lesions on MRI.

Conclusions: Cognitive impairment was ubiquitous in this sample of active NCYST patients. Antiepileptic drug use and seizure frequency could not account for these features. Dementia was present in a significant proportion of patients. These data broaden our knowledge on the clinical presentations of NCYST and its impact in public health.

Keywords: neurocysticercosis, cognition, neurobehavioral manifestations, dementia, epilepsy. 


\section{INTRODUÇÃO}

\subsection{Parasitologia}

O homem é o único hospedeiro da forma adulta do parasita Taenia solium. No ciclo biológico do parasita, o suíno ingere os ovos eliminados com as fezes humanas, devido aos hábitos coprofágicos. Uma vez ingeridos, os ovos liberam os embriões hexacantos no sistema digestório dos suínos, que migram ativamente para a circulação sanguínea, fixando-se em tecidos de sua predileção, como o tecido muscular, a tela subcutânea e o sistema nervoso central (SNC). Nesses locais, o embrião desenvolve uma vesícula característica, o Cysticercus cellulosae, com o escólex invaginado, que por sua vez constitui a forma larvária da Taenia solium.

Ao ingerir carne suína mal cozida e contaminada com cisticercos, o homem desenvolve a teníase, que atinge a fase de verme adulto no intestino delgado proximal. A Taenia cresce a partir da região proximal, o colo, e desprende anéis na região terminal, repletos de ovos que se disseminam no meio ambiente pelas fezes humanas. A ingestão dessas fezes por porcos completa o ciclo de vida do parasita. A cisticercose ocorre quando o ser humano ingere ovos da Taenia, desempenhando o papel de hospedeiro intermediário que no ciclo natural é reservado aos suínos (Canelas, 1962; Vaz, 1996; Garcia, 2005).

Estima-se que $5 \%$ a $40 \%$ dos pacientes com teníase possuem quadro concomitante de cisticercose. Desta forma, a neurocisticercose (NC) é causada pela presença da forma larvária da Taenia solium no SNC. Cada cisticerco sobrevive cerca de3 a 6 anos, e evolui para degeneração espontânea após esse período (Vaz, 1996; Takanayagui, 1983). 


\subsection{Epidemiologia}

A NC é a doença parasitária mais frequente do SNC em adultos. É altamente prevalente em países da América Central, do Sul, Ásia e África (Roman et al, 2000; Carpio, 2002; Varma et al, 2002; Agapejev , 2003; Del Brutto et al, 2004; Kraft et al, 2007). No entanto, nas últimas décadas, um número crescente de casos foram relatados nos Estados Unidos (Shandera et al, 1994; Ong et al, 2002; Townes et al, 2004; De La Garza et al, 2005) e em países da Europa (Alvarez-Rodríguez, 2005; Barra-Valencia et al, 2007; Más-Sesé et al, 2008).

A Organização Mundial da Saúde (OMS) calcula que ocorram cerca de 50 mil mortes ao ano devido à NC (Roman et al, 2000). Um número muito maior de indivíduos apresenta complicações crônicas não fatais da NC, como epilepsia e síndrome de hipertensão intracraniana, causando impacto negativo sobre a qualidade de vida dos habitantes e sobre a economia nas áreas endêmicas. Em nosso meio, onde uma grande parte da população está exposta à Taenia solium, um estudo de necropsias mostrou prevalência de 1,5\% de NC (Agapejev, 2003).

\subsection{Quadro Clínico}

As apresentações clínicas da NC são muito variáveis, podendo se manifestar em diferentes níveis de gravidade. O quadro clínico depende da interação do parasita com os fatores de defesa do hospedeiro, variando de acordo com o tipo 
morfológico do cisticerco, número de cistos, sua localização e fase de desenvolvimento do parasita:

1.3.1 Tipo morfológico: a forma Cysticercus cellulosae dá origem à forma vesicular clássica, em geral intraparenquimatosa, sendo a mais prevalente na maioria das casuísticas mundiais. Diferente desta forma vesicular, a forma racemosa (Cysticercus racemosus) possui $40 \mathrm{~mm}$ a $120 \mathrm{~mm}$ de diâmetro; habitualmente é desprovida de escólex e é formada por múltiplas vesículas confluentes, sendo mais comum nas cisternas da base do crânio. É discutível se a forma racemosa corresponde à mesma espécie parasitária ou se apenas consiste em variante relacionada à presença do parasita no espaço continente do líquido cefalorraquidiano (LCR). Possui este nome por ter suas formações semelhantes a cachos de uva (Vaz, 1996; Garcia, 2005).

1.3.2 Número de cistos: pode haver desde um cisto isolado, forma mais comum em alguns países como a Índia, por exemplo, até várias centenas de cisticercos.

1.3.3 Localização dos cistos: podem localizar-se no espaço subaracnoideo da convexidade do cérebro, nas cisternas, no parênquima (neste caso sendo mais comum na transição córtico-subcortical), nos ventrículos e no canal raquiano.

1.3.4 Fase de evolução biológica do parasita:

a) Inicialmente os cistos são biologicamente viáveis, apresentando-se morfologicamente como vesículas íntegras, sem qualquer evidência de processo inflamatório ao seu redor. Isto ocorre graças à indução de imunodepressão local e à ativação de mecanismos de evasão imunológica (Garcia et al, 2005). É o estágio vesicular: o fluido vesicular é claro e o 
escólex aparece como estrutura bem individualizada. Há uma situação de equilíbrio imunológico entre o parasita e o hospedeiro, que permanece assintomático ou oligossintomático.

b) Após um período que pode variar de 3 a 6 anos, ou após o tratamento com drogas parasiticidas, os cistos sofrem degeneração. No início desse processo, o fluido vesicular se torna turvo e o escólex passa a apresentar sinais de degeneração. É o estágio coloidal. Uma vez exposta a superfície do parasita ao sistema imunológico do hospedeiro, há perda dos mecanismos de supressão e ocorrem degeneração do cisto e rotura de barreira hemato-encefálica localmente. O parasita passa a apresentar cápsula espessa; o tecido ao redor apresenta edema e ativação astrocítica com reação gliótica periférica. Aos exames de neuroimagem, pode-se observar nessas situações o aparecimento de realce anelar à tomografia computadorizada (TC) de crânio ou hipersinal em imagens ponderadas em T2 ou FLAIR (“Fluid-attenuated Inversion Recovery Image”) à RM (Takanayagui, 1983, 2001; Del Brutto et al, 2001: Lucato et al, 2007).

c) Após o período inicial de inflamação, desenvolve-se o estágio nodular, em que o conteúdo do cisto torna-se ainda mais espesso e o escólex deixa de ser bem caracterizado. O cisto assume forma nodular, que pode ser confundida com imagens de granulomas ou de tumores metastáticos. Nesta fase persiste a reação inflamatória peri-lesional.

d) A seguir, há redução importante do tamanho do cisto, atingindo apenas cerca de $20 \%$ do tamanho original. Gradualmente passa a haver deposição de sais de cálcio até que o cisto atinja a fase de grânulo calcificado. 
Esse último estágio é fonte de grande discussão na literatura atual. Anteriormente se admitia que a fase calcificada fosse completamente inativa, inerte, cicatricial, sem atividade antigênica. Entretanto, com elevada frequência, observam-se, à RM realces inflamatórios peri-lesionais em formas calcificadas. Esse achado intrigante levou alguns autores a admitirem a possibilidade teórica de que embora calcificados, ao menos na sua fase inicial esses cistos residuais possam continuar apresentando atividade imunogênica. Tal hipótese explicaria a persistência de manifestações clínicas de epilepsia em alguns pacientes durante períodos variáveis após a caracterização da calcificação dos parasitas (Carpio, 2002; Garcia et al, 2004; Sotelo et al, 1996, 2004; Nash et al, 2004).

É surpreendente constatar que a maioria dos estudos sobre manifestações clínicas da NC não fornece informações quanto ao estágio de evolução dos parasitas nos pacientes estudados, não deixando clara a porcentagem da sua casuística formada por pacientes com a forma calcificada (cicatricial), pela forma coloidal/nodular (em degeneração) e a vesicular (oligo/assintomática) (Boppré et al, 2001; Ramirez-Bermudez et al, 2005; Forlenza et al, 1997, 1998; Terra-Bustamante et al, 2005). Em áreas endêmicas há uma grande parte da população com infestação resolvida espontaneamente e que talvez nunca tenha apresentado sintomatologia exuberante. Cerca de $20 \%$ de indivíduos assintomáticos residentes em áreas endêmicas possuem calcificação intracraniana (Garcia et al, 2005; Roman et al, 2000; Medina et al, 2005). Assim, estudos que não explicitam o estágio da doença de seus pacientes podem estar avaliando pacientes com NC resolvida, completamente assintomáticos, que apresentam alterações clínicas não relacionadas à $\mathrm{NC}$, e 
sim a outras afecções igualmente prevalentes na população geral. Por exemplo, outras causas de epilepsia, outras doenças infecciosas e transtornos do humor, e do funcionamento cognitivo não relacionados à NC.

Analisando uma casuística de 276 pacientes com NC, Canelas (1962) dividiu as apresentações clínicas da doença da seguinte forma:

- Forma hipertensiva: há componente de Síndrome de Hipertensão Intracraniana (HIC). Na maioria das vezes é associada ao bloqueio do fluxo liquórico, estando mais frequentemente relacionada com a forma racemosa da doença;

- Forma convulsiva: encontrou-se igual prevalência de crises focais e generalizadas. Esta forma foi a mais prevalente nesta casuística avaliada, juntamente com HIC;

- Formas com manifestações neurológicas focais ou difusas: manifestações neurológicas isoladas, não associadas a formas hipertensivas ou convulsivas, foram encontradas em menos de $10 \%$ dos pacientes;

- Forma psíquica: apesar de bem caracterizada nesta casuística, foram encontradas somente em associação a outras manifestações clínicas como HIC, crises convulsivas ou manifestações neurológicas generalizadas da doença. O autor não encontrou casos isolados de alterações psiquiátricas na casuística avaliada.

Em estudo mais recente, as manifestações clínicas mais frequentemente observadas na NC foram crises epilépticas (62\%), HIC (38\%), meningite 
cisticercótica $(35 \%)$ e transtornos psiquiátricos (11\%). Mais raramente, os pacientes apresentam forma apoplética ou endarterítica $(2,8 \%)$ e síndrome medular (0,5\%) (Takanayagui et al, 1983, 2001).

\subsection{Diagnóstico da NC}

Há alguns anos foram propostos critérios para o diagnóstico da NC (Del Brutto et al, 2001). Estes critérios são divididos em: absolutos, maiores, menores e epidemiológicos. O diagnóstico é denominado definitivo ou provável com base em combinações dos seguintes critérios:

a) Critérios absolutos:

1. Demonstração histológica do parasita por biópsia de cérebro ou medula espinhal;

2. Lesão cística com evidência do escólex em TC ou RM;

3. Visualização subretiniana direta do parasita à fundoscopia.

b) Critérios maiores:

1. Lesões altamente sugestivas de $\mathrm{NC}$ em estudos de neuroimagem;

2. Anticorpos anticisticercos séricos positivos ("enzime-linked immunotransfer blot" - ELISA);

3. Resolução de lesão cística intracraniana após tratamento com albendazol ou praziquantel; 
4. Resolução espontânea de lesão única pequena captante de contraste em exame de neuroimagem.

c) Critérios menores:

1. Lesões compatíveis com NC em estudos de neuroimagem;

2. Manifestação clínica sugestiva de NC;

3. Reação de ELISA no LCR positiva para anticorpos anticisticerco ou antígenos de cisticercos.

d) Critérios epidemiológicos:

1. Evidência de contactante doméstico com teníase;

2. Indivíduos naturais ou provenientes de região onde NC é endêmica;

3. História de viagem frequente para localidades em que NC é endêmica.

- Diagnóstico definitivo:

Presença de 1 critério absoluto;

Presença de 2 critérios maiores, 1 menor e 1 epidemiológico.

- Diagnóstico provável:

Presença de 1 critério maior e 2 menores;

Presença de 1 critério maior, 1 menor e 1 epidemiológico; 
Presença de 3 critérios menores e 1 epidemiológico.

A publicação que propõe estes critérios diagnósticos faz referência a certos achados de neuroimagem relacionados à NC (Del Brutto et al, 2001). No item "Critérios maiores", os autores se referem a lesões "altamente sugestivas de NC". Este tipo de lesão é explicitado no próprio texto como:

a. Lesões císticas sem visualização do escólex;

b. Lesões realçadas com contraste, tanto anelares como nodulares, únicas ou múltiplas;

c. Calcificações arredondadas intraparenquimatosas.

As lesões descritas nos "Critérios menores" como "compatíveis com NC" são as seguintes:

a. Hidrocefalia;

b. Realce anômalo nas leptomeninges;

c. Mielograma evidenciando múltiplas falhas de enchimento.

Atualmente, essa proposta de critérios diagnósticos é popular e bastante difundida. No entanto, pacientes bem heterogêneos podem preencher os critérios diagnósticos para NC. Isto ocorre porque tais recomendações privilegiam os dados de neuroimagem em detrimento da apresentação clínica da NC. Isso faz com que sejam selecionados preferencialmente pacientes com a forma cística da doença, deixando de lado aqueles que apresentam 
manifestações clínicas da forma racemosa da NC, como HIC. Assim, ao se utilizar estes critérios acaba-se por incluir um maior número de pacientes com as formas convulsivas da NC, mais associadas a cistos intracranianos (Garcia et al, 2005; Vaz, 1996; Montano et al, 2005; Bragazza et al, 2002).

É preciso ainda chamar atenção para o fato de que as reações sorológicas qualificadas como "critério maior" indicam somente que houve contato do indivíduo com o parasita. E que nestes pacientes, a positividade indica apenas infestação pela $T$. solium, não sendo possível identificar sua forma, se teníase ou cisticercose. Ainda no caso de cisticercose, ela pode estar presente em outros tecidos, como nos músculos e olhos, não sendo exclusiva do SNC, levando à positividade da sorologia em todos estes casos.

Assim, estudos que desejam se aprofundar nas manifestações clínicas da NC não devem se restringir apenas à seleção de pacientes por "critérios diagnósticos para NC", mas atentar para o fato que mesmo dentro dos critérios acima existem pacientes em fases diferentes da evolução da doença, sujeitos a diferentes complicações e de prognóstico diverso.

Pesquisas sobre essa doença devem definir claramente que tipo de pacientes com NC está se avaliando e particularizar seus achados para cada um destes diferentes subtipos. A seleção do grupo controle também deve privilegiar estas nuances relacionadas à NC.

\subsection{Quadro cognitivo}

As alterações neuropsiquiátricas relacionadas à NC foram relatadas inicialmente por Griesinger, em 1862. Outros, como Arriagada (1961), voltaram a contribuir com o tópico na metade do século passado, propondo 
inclusive uma classificação da síndrome neuropsiquiátrica (Ramirez-Bermudez et al, 2005).

Diversos estudos retrospectivos foram publicados nas últimas décadas. O estudo de Agapejev (2003) chama atenção porque relata a frequência dessas alterações em $9 \%$ a $23 \%$ dos pacientes. Canelas (1962) encontrou essas alterações somente associadas à HIC, déficits neurológicos focais e síndrome convulsiva.

Nas últimas duas décadas foram publicados os primeiros estudos prospectivos sobre o tema. Em 1997, Forlenza et al., 1997 avaliaram 38 pacientes com NC, utilizando duas escalas de avaliação do estado mental. Com o uso do MiniExame do Estado Mental (MEEM) encontraram apenas dois pacientes com pontuações abaixo do valor esperado para o seu nível educacional. Já com o uso da escala Exame do Estado Mental (EEM) de Strub e Black, os autores encontraram alterações neuropsiquiátricas moderadas ou leves em $71,9 \%$, e graves em $15,6 \%$ dos doentes. Todos os pacientes avaliados por esse método apresentavam déficit de atenção. Utilizando escalas para depressão, Forlenza encontraram prevalência de 52,6\% desta condição na sua casuística. Apesar de terem inicialmente dividido os pacientes em subgrupos com forma ativa da doença (com presença de cistos) ou inativa (com calcificações), os autores não correlacionaram seus achados com estas variáveis.

Este estudo é de grande importância por ter inaugurado uma nova etapa de pesquisa de alterações neuropsiquiátricas em NC, utilizando avaliações estruturadas com instrumentos bem validados e específicos, além de ter sido o primeiro estudo prospectivo sobre o tema. No entanto, apresenta algumas limitações metodológicas. Os autores não utilizaram grupo controle. Como 
$66 \%$ dos seus pacientes tinham escolaridade menor que 4 anos, as alterações nos testes neuropsicológicos podem ser, em parte, devido a uma situação prémórbida decorrente do nível educacional baixo. O uso de controles contornaria esta questão, pois o desempenho dos doentes com NC poderia ser comparado a controles saudáveis com nível educacional semelhante. Além disso, a alta prevalência de depressão na amostra dificulta a interpretação dos achados. Isto ocorre porque tanto a depressão quanto o uso de drogas antiepilépticas (DAE) causam déficits atencionais o que interfere no funcionamento de outros domínios cognitivos, e que foram encontrados em $100 \%$ de seus pacientes.

Dois estudos adicionais avaliaram o perfil cognitivo de pacientes com $\mathrm{NC}$ e epilepsia: o de Boppré et al (2001) e o de Terra-Bustamante et al (2005); o primeiro comparou três grupos de indivíduos: um grupo de 22 pacientes com epilepsia sintomática secundária a diversas etiologias; um grupo de 20 pacientes com epilepsia sem causa aparente (a não ser presença de calcificações intracranianas sugestivas de NC prévia); e um grupo controle, sem epilepsia, composto de 29 indivíduos. O estudo não encontrou diferenças entre os grupos de doentes epilépticos com e sem calcificações intracranianas sugestivas de NC. Ambos os grupos tiveram desempenho inferior ao grupo controle sem epilepsia em testes de avaliação de memória verbal, reconhecimento espacial, habilidades visuais-construtivas, reconhecimento de faces famosas, abstração e julgamento. Os autores não relataram a presença de depressão nos pacientes. Estes dados são uma valiosa contribuição ao tema. No entanto, o estudo se restringiu a pacientes que apresentavam calcificações intracranianas associadas à epilepsia, uma população que poderia nem receber 
o diagnóstico de $\mathrm{NC}$ se com eles fossem utilizados os critérios atuais (Del Brutto et al, 2001).

O segundo estudo (Terra-Bustamante et al, 2005) avaliou uma população de pacientes com epilepsia sintomática secundária à esclerose mesial temporal (EMT) composta por portadores $(n=32)$ e não portadores $(n=48)$ de calcificações intracranianas, e da mesma forma que o anterior, não encontrou diferenças na avaliação cognitiva entre os dois grupos. No entanto, este estudo utilizou somente pacientes com EMT e que foram submetidos à ressecção cirúrgica,avaliando, assim, um grupo bem restrito de indivíduos. Seu maior valor talvez seja o de ampliar o conhecimento sobre o papel de calcificações intracranianas associadas a outras causas de epilepsia sintomática e a influência desta associação no prognóstico pós-operatório dos doentes com EMT.

Outro estudo sobre alterações cognitivas em NC foi publicado posteriormente (Ramirez-Bermudez et al, 2005). Os autores avaliaram 78 pacientes com diagnóstico de NC sem tratamento. De acordo com os critérios do DSM-IV, eles encontraram quadro de demência em 14 indivíduos. Os pesquisadores relatam que após o tratamento com drogas antiparasitárias, 11 pacientes apresentaram melhora cognitiva, não mais preenchendo critérios diagnósticos para demência. Nesse estudo a população considerada com demência foi diferente em muitos aspectos da população sem demência. O grupo com demência tinha média de idade quase duas vezes maior que o sem demência; também possuía média de 2 anos de escolaridade, enquanto o grupo sem demência apresentava média de 9 anos. Outra grande diferença foi a prevalência de epilepsia entre esses dois grupos. O grupo com demência apresentava epilepsia em 12 dos 14 pacientes (85\%). Já o grupo sem demência 
apresenta epilepsia em 58\% dos casos. Estes valores levantam dúvida se variáveis como idade, nível educacional e presença de epilepsia associada, variáveis comprovadamente relacionadas ao comprometimento cognitivo (Bertolucci et al, 1994; Lefèvre , 1969; Singh et al, 2004; Sachdev et al, 2004), não estariam influenciando o desempenho nos testes utilizados e, dessa forma, superestimando um provável efeito da doença de base sobre o quadro cognitivo. Também chama atenção o fato de não se ter avaliado a presença de depressão nessa casuística, uma vez que esta é uma afecção presente em grande parte dos pacientes com NC e é sabidamente associada a alterações cognitivas (Bertelson e Price, 2003).

Outra limitação é a ausência de avaliação da presença de abuso ou dependência de álcool nas amostras estudadas. Trata-se de condição frequente em populações de baixa renda e baixo nível de escolaridade, assim como a $\mathrm{NC}$, podendo também causar alterações cognitivas em seus usuários, seja por abstinência, pelo uso crônico ou por deficiências nutricionais relacionadas.

Em seu estudo, Ramirez-Bermudez et al (2005) dividiram seus pacientes quanto à forma de $\mathrm{NC}$ em um grupo apresentando a forma ativa (vesículas) e inativa (calcificações). No entanto, não dizem quantos casos com forma ativa apresentavam degeneração dos cistos. Tal informação é importante, uma vez que esses pacientes apresentam processo inflamatório ativo intracraniano, devendo ser avaliados separadamente dos demais por estarem sujeitos a complicações que podem confundir a interpretação da avaliação cognitiva, como delirium e HIC. Da mesma forma, não se revelou se os pacientes considerados "curados" de sua demência após o tratamento antiparasitário (11 entre os 14 iniciais) possuíam lesões ativas ou inativas. Essa informação seria 
desejável, pois enriqueceria o conhecimento da fisiopatologia das alterações cognitivas relacionadas à NC.

Apesar do comprometimento cognitivo associado à $\mathrm{NC}$ ser reconhecido como uma das manifestações mais frequentes da doença (Sotelo et al, 1985; Carpio et al, 2002), os seus detalhes permanecem pobremente caracterizados, sendo certamente subdiagnosticada na prática clínica. Isso se deve em parte à absoluta falta de estudos controlados sobre o assunto, utilizando ferramentas de avaliação cognitiva validadas. Os poucos estudos dedicados à análise das repercussões neuropsiquiátricas da $\mathrm{NC}$ sugeriram que algumas de suas manifestações, como a demência, podem ser mais frequentes do que inicialmente acreditado (Forlenza et al, 1997; Ramirez-Bermudez et al, 2005). No entanto, a generalização destas conclusões é limitada pela falta de grupos controles e critérios que distingam sub-formas da $\mathrm{NC}$, como a ativa e inativa.

Este é o primeiro estudo controlado sobre as manifestações neuropsiquiátricas da $\mathrm{NC}$ em pacientes sem tratamento, apresentando exclusivamente $\mathrm{NC}$ em sua forma ativa, definida por critérios absolutos (Del Brutto et al, 2005) pela presença de ao menos um cisto com escólex íntegro à $\mathrm{RM}$ de crânio. $\mathrm{O}$ desempenho cognitivo e funcional de pacientes com NC foi comparado ao de controles saudáveis (CS) devidamente emparelhados por idade e nível educacional. Ainda, com o intuito de aferir se a presença de crises epilépticas e uso de DAE poderia influenciar o desempenho cognitivo destes pacientes, comparamos pacientes com NC a pacientes com epilepsia criptogênica (EC) de similar idade, nível educacional, frequência de crises epilépticas e padrão de uso de DAE. 


\section{OBJETIVOS}

\subsection{Objetivo principal}

Avaliar o desempenho de pacientes com NC ativa em testes de avaliação cognitiva e funcional em comparação a controles saudáveis e a pacientes com epilepsia criptogênica.

\subsection{Objetivos específicos}

1) Avaliar a frequência de demência e de comprometimento cognitivo sem demência em pacientes com NC ativa;

2) Analisar o perfil de alterações cognitivas em pacientes com NC ativa;

3) Investigar a correlação entre os achados à RM de crânio, os escores em testes cognitivos e a frequência de demência em pacientes com NC ativa. 


\section{MÉTODOS}

O estudo foi realizado na Clínica Neurológica do Hospital das Clínicas da Faculdade de Medicina da Universidade de São Paulo (HCFMUSP) com envolvimento direto dos Ambulatórios de Neuroinfectologia, Neurologia Cognitiva e do Comportamento, e Epilepsia.

O projeto de pesquisa foi aprovado pela Comissão de Ética para Análise de Projetos de Pesquisa (CAPPesq) do HCFMUSP. Todos os participantes foram claramente esclarecidos quanto aos objetivos da pesquisa e do caráter voluntário de sua participação, e assinaram o Termo de Consentimento Livre e Esclarecido.

\subsection{Participantes}

Todos os participantes foram avaliados por um neurologista devidamente treinado na administração de testes cognitivos e que desconhecia a qual grupo de estudo pertencia cada indivíduo. Tal avaliação incluiu anamnese, triagem de abuso de álcool através do questionário CAGE e AUDIT (Ewing, 1984; Saunders et al, 1993) e coleta de exames laboratoriais para avaliação de causas secundárias de déficit cognitivo e demência - eletrólitos, função tireoidiana, níveis de vitamina B12 e ácido fólico, hemograma completo e testes imunológicos para infecção pelo HIV e Sífilis (Nitrini et al, 2005).

$\mathrm{O}$ tipo de manifestação clínica inicial dos pacientes com NC foi registrada de acordo com os dados de prontuário, ou através da anamnese. Os diferentes 
tipos de apresentação clínica foram organizados de acordo com Canelas (1962), incluindo:

a) Crise convulsiva seguida de síndrome epiléptica sintomática;

b) Síndrome de HIC: definida pela síndrome clínica (cefaleia, náuseas e vômitos, papiledema, alteração do nível de consciência);

c) Crise convulsiva sintomática aguda;

d) Déficit neurológico focal;

e) Cefaleia isolada sem sinais de HIC.

A duração da doença em meses e a principal síndrome clínica atual também foram registradas.

Indivíduos dos grupos EC e CS (descrição abaixo), apresentando alterações na triagem de abuso de álcool ou nos exames sanguíneos, foram excluídos do estudo. A presença de altos níveis de colesterol, hipertensão arterial sistêmica, diabete mélito, disfunção tireoidiana, sintomas depressivos, acidente vascular encefálico e antecedente de traumatismo craniano foi investigada em todos os participantes.

\subsection{Pacientes com NC}

Pacientes com idade inferior a 60 anos, sem tratamento específico, preenchendo critérios definitivos de NC em sua forma ativa (Sotelo et al, 1985) foram incluídos neste grupo. NC definida foi diagnosticada por meio de 
critérios absolutos, segundo Del Bruto et al (2001), ou seja, pela presença de ao menos uma lesão cística com escólex à RM de crânio. Assim, um primeiro exame de RM foi utilizado para determinar a presença de $\mathrm{NC}$ em sua forma ativa. Esse mesmo exame permitiu a aferição da carga de lesões de NC através da contagem do número de lesões, seu tipo (cisto com ou sem escólex, cisto com edema, cistos transicionais, lesões calcificadas e lesões racemosas), localização geral (hemisfério direito/esquerdo, cortical/subcortical, ventrículo lateral/terceiro/quarto ou fossa posterior) e lobo cerebral acometido (frontal, parietal, temporal ou occipital). As imagens foram avaliadas por neurorradiologistas que desconheciam os resultados dos testes cognitivos dos pacientes (Lucato et al, 2007).

Como na maior parte dos casos, a avaliação cognitiva não foi realizada no mesmo dia da visita de inclusão, mas sim algumas semanas ou, eventualmente, alguns meses após; todos os pacientes foram submetidos a uma segunda RM ou TC de crânio 15 dias antes ou 15 após a avaliação cognitiva, com o intuito de se confirmar que uma lesão com escólex ainda estava presente no momento da avaliação cognitiva (Ramirez-Bermudez et al, 2005). Dessa forma, ficou assegurado que não havia ocorrido degeneração de cistos naquele período, o que poderia influenciar o desempenho cognitivo devido à presença de delirium ou encefalopatia (Forlenza et al, 1999).

\subsection{Grupos de comparação}

Pacientes com EC seguidos em nossa instituição foram submetidos à avaliação cognitiva. EC foi diagnosticada pela presença de crises epilépticas com 
características semiológicas e eletroencefalográficas focais em presença de RM normal, de acordo com os critérios da ILAE (International League Against Epilepsy, 1989). Todos os pacientes apresentavam exames de RM normais, incluindo uma delas realizada durante os dois anos precedendo a inclusão no estudo, além de todos os exames de EEG, apresentando descargas epilépticas focais. Pacientes com quadro clínico ou eletroencefalográfico sugestivo de esclerose mesial temporal foram excluídos, mesmo em vigência de RM normal.

Pacientes com antecedentes de infecção intestinal crônica ou teníase (autorrelato) também foram excluídos do estudo. Todos os pacientes do grupo EC foram submetidos a exame de TC para se avaliar, especificamente, a presença de calcificações. Pacientes apresentando calcificações intracranianas foram excluídos (Terra-Bustamante et al, 2005; Velasco et al, 2006; Bianchin et al, 2006).

CS foram recrutados entre os funcionários de nossa instituição e parentes de pacientes hospitalizados em outras enfermarias do hospital. Para serem incluídos, deveriam ter idade inferior a 60 anos, ausência de antecedentes de epilepsia, de infecções intestinais crônicas ou doença neurológica, além de exame de TC de crânio estritamente normal. As análises de neuroimagem foram realizadas por um neurologista e neurorradiologistas que desconheciam os resultados dos exames cognitivos e de avaliação funcional.

Todos os participantes do estudo foram avaliados quanto à presença de depressão, segundo os critérios do DSM-IV. Indivíduos deprimidos do grupo CS foram excluídos do estudo. Aqueles dos grupos $\mathrm{NC}$ e EC foram 
inicialmente tratados com fluoxetina $20 \mathrm{mg}$ ao dia e reavaliados em consultas mensais. Participantes com depressão só foram submetidos à avaliação cognitiva uma vez que se apresentassem assintomáticos por um período mínimo de 2 meses consecutivos.

Os pacientes dos grupos NC e EC (tanto aqueles originalmente sem depressão como os que responderam ao seu tratamento) foram avaliados somente se apresentassem:

1) Ausência de crises epilépticas por ao menos duas semanas precedendo a avaliação cognitiva;

2) Tratamento em dose fixa de DAE por ao menos um mês precedendo a avaliação;

3) Ausência de sinais clínicos de intoxicação de DAE (níveis séricos de DAE foram mensurados em casos de suspeita clínica);

4) Ausência de sinais de delirium segundo uma escala de avaliação específica (Delirium Rating Scale; Trzepacz et al, 1988).

Pacientes que não preenchiam todos estes critérios tiveram sua avaliação cognitiva adiada por 2 meses e realizaram um terceiro exame de RM com o intuito de excluir a presença de cistos em degeneração.

3.4 Recrutamento e emparelhamento 
Com base em estudos prévios, considerou-se que 50 pacientes com $\mathrm{NC}$ ativa seriam suficientes para testar a nossa hipótese (Forlenza et al, 1999 ; RamirezBermudez et al, 2005).

O emparelhamento foi realizado de forma liberal, em blocos de 10 pacientes: para cada 10 pacientes com NC incluídos, idade e nível educacional foram estratificados, sendo que esta informação foi usada para o recrutamento do próximo bloco de 10 indivíduos com EC e CS. As estratificações para idade e nível educacional tiveram intervalos de 5 e 4 anos, respectivamente.

Devido ao aumento da quantidade de pacientes procurando o nosso serviço, e já tendo recebido tratamento antiparasitário durante a fase final do estudo, o recrutamento diminuiu sensivelmente no último terço do período de coleta de dados. Assim, a seleção de pacientes do grupo NC terminou após a inclusão do $45^{\circ}$ paciente.

Os prontuários de 51 pacientes com EC foram analisados no ambulatório de Epilepsia do HCFMUSP e equiparados por idade, nível educacional e frequência de crises epilépticas (menos de 1/mês, 1-3/mês, 1/semana, mais de uma por semana). Enquanto os pacientes do grupo $\mathrm{NC}$ apresentaram baixo nível educacional, pacientes com EC apresentaram escolaridade mais elevada. Isso levou ao não recrutamento de diversos potenciais participantes, fazendo com que esse grupo apresentasse somente 28 integrantes ao fim do estudo.

\subsection{Avaliação cognitiva e funcional}


Todos os participantes foram submetidos à avaliação cognitiva com os seguintes instrumentos: mini-exame do estado mental (MEEM) (Folstein, 1975; Brucki et al, 2003), extensão de dígitos em ordem direta (EDD) e inversa (EDI) (Wechsler, 1981), desenho do relógio (DR), evocação da lista de 10 palavras (memória verbal - MV), praxia construtiva (PC), teste de fluência verbal semântica (FVS), teste de nomeação (TN) e teste de trilhas A (TTA) e B (TTB) da "Comprehensive Battery of the Consortium to Establish a Registry of Alzheimer's Disease” (CERAD) (Morris, 1989; Bertolucci et al, 2001); bateria breve de rastreio cognitivo (BBRC) (Nitrini et al, 1994, 2004); e o teste de interferência de cores de Stroop (TICS) (Stroop, 1935).

O desempenho funcional foi avaliado pelo Questionário de Atividades Funcionais de Pfeffer (PFAQ; Pfeffer, 1982), preenchido a partir da entrevista com um acompanhante ou familiar próximo que tivesse contato frequente com o indivíduo. Escores acima de 5 foram considerados como indicativos de comprometimento funcional.

Demência foi diagnosticada de acordo com os critérios do DSM-IV, os quais foram operacionalizados da seguinte forma:

1) Escores abaixo do Intervalo de Confiança (IC) $95 \%$ dos valores obtidos pelos CS para os testes de evocação tardia da bateria do CERAD (MV) e da BBRC;

2) Escores abaixo do IC 95\% dos valores obtidos por CS em pelo menos um teste adicional relacionado a um segundo domínio cognitivo além da memória;

3) Comprometimento funcional de acordo com a pontuação no PFAQ. 
O comprometimento cognitivo sem demência (CCSD) foi diagnosticado nos pacientes preenchendo todos os critérios de demência acima, com exceção de prejuízo funcional avaliado pelo PFAQ.

\subsection{Análise estatística}

Todos os valores foram expressos em médias e desvios-padrão, salvo indicação específica em contrário. O teste de Kolmogorov-Smirnov foi utilizado para se aferir a normalidade dos valores obtidos nos testes cognitivos. Assim, os dados não paramétricos foram comparados a partir do teste de Kruskal-Wallis, seguido pelo teste de Mann-Whitney, quando indicado. Análises de correlação foram realizadas pelo teste de Correlação de Spearman. Variáveis com distribuição normal, como os dados clínicos dos pacientes, foram comparados a partir do teste t de Student bicaudal.

Os escores cognitivos de cada indivíduo do grupo $\mathrm{NC}$ foram comparados aos de CS. Resultados abaixo do IC 95\% foram considerados alterados, a não ser pelos testes cronometrados (TTA, TTB, e TICS), em que escores acima do IC 95\% foram considerados anormais. Variáveis categóricas foram comparadas através do Teste Exato de Fisher. As análises inferenciais e correlações foram realizadas com o programa Statview, em sua versão 4.0.

A análise para o cálculo do poder do estudo foi realizada com os escores do MEEM, por se tratar de um teste de avaliação cognitiva global (multidimensional), comparando-se os grupos NC e EC. Para o cálculo do poder do estudo, utilizou-se o programa Power and Sample Calculations, em sua $3^{\mathrm{a}}$ versão. 


\section{RESULTADOS}

\subsection{Pacientes excluídos da análise}

Foram incluídos no grupo NC 45 pacientes; 5 pacientes do grupo NC não foram submetidos à avaliação cognitiva devido à presença de retardo mental $(n=1)$, depressão refratária $(n=1)$ e por terem apresentado degeneração do último cisto íntegro com escólex no intervalo de tempo entre a primeira RM (de inclusão) e a segunda, realizada próxima da avaliação cognitiva e, desta forma, não mais apresentando $\mathrm{NC}$ definida por critérios absolutos (n=3).

\subsection{Características gerais dos participantes}

40 pacientes com NC ativa, 28 pacientes com EC e 49 CS foram submetidos à avaliação cognitiva, funcional e de neuroimagem completa, tendo sido assim incluídos na análise estatística.

Participantes dos três grupos (NC, EC, CS) apresentaram semelhanças em relação à idade $(39,2 \pm 10,5$ anos; $39,3 \pm 12,5 ; 39,4 \pm 12,7$; respectivamente, $p$ $=0,89$ e $\mathrm{p}=0,91)$ e nível educacional $(6,2 \pm 4,0 ; 7,3 \pm 3,5 ; 8,1 \pm 3,0$ anos; respectivamente, $p=0,20$ e $p=0,19)$ (TABELA 1).

Os pacientes do grupo NC tiveram como origem estados das regiões Nordeste (50\%), Sudeste (40\%), Centro-Oeste (5\%) e Sul (5\%). Por ocasião do estudo, 87,5\% dos participantes residiam no Estado de São Paulo.

Excluindo-se os resultados da avaliação cognitiva, a manifestação clínica inicial mais frequente na $\mathrm{NC}$ foi epilepsia $(67,5 \%)$, HIC $(27,5 \%)$, crise 
convulsiva sintomática aguda (17,5\%), déficit neurológico focal (10\%) e cefaleia isolada sem sinais de HIC (2,5\%). Todos os pacientes incluídos no estudo apresentavam síndrome epiléptica sintomática no momento da avaliação; 10 pacientes (25\%) apresentaram mais de uma manifestação clínica. O tempo médio decorrido entre o primeiro sintoma de $\mathrm{NC}$ e a avaliação cognitiva foi de 7 anos e 3 meses.

Pacientes do grupo EC apresentaram frequência mensal de crises epilépticas $(4,8 \pm 1,7)$ e número médio de DAE $(1,5 \pm 0,6)$ estatisticamente semelhantes ao grupo $\mathrm{NC}(5,4 \pm 2,3$ e $1,1 \pm 0,4$, respectivamente; $p=0,109$ e $p=0,581$ Teste $\mathrm{t}$ de Student bicaudal). A distribuição do tipo de DAE utilizado por integrantes dos grupos EC e NC também foi estatisticamente semelhante: carbamazepina foi usada por $62,5 \%$ dos pacientes com NC e por $65,5 \%$ daqueles com EC $(p=0,35)$; fenobarbital foi usado por $25,8 \%$ do grupo $\mathrm{NC}$ e por $17,2 \%$ do grupo EC $(p=0,42)$; e fenitoína foi utilizada por $18,7 \%$ dos pacientes com $\mathrm{NC}$ e por $10,3 \%$ daqueles com EC ( $\mathrm{p}=0,80$, Teste Exato de Fisher) (TABELA 1).

Níveis de colesterol sérico, presença de hipertensão arterial sistêmica, diabete mélito, antecedente de traumatismo craniano, disfunção tireoidiana, sintomas depressivos e acidente vascular encefálico foram semelhantes entre integrantes dos três grupos de estudo $(\mathrm{p}>0.4$ para todos os testes, teste qui quadrado. Nenhum paciente no grupo $\mathrm{NC}$ apresentou escores alterados nos questionários CAGE e AUDIT. 


\subsection{Presença de depressão}

Depressão esteve presente em $11(27,5 \%)$ pacientes com NC; foi tratada com sucesso em $10(90,9 \%)$ deles. Assim, 40 pacientes com NC definida por critérios absolutos em forma ativa e sem tratamento específico para NC foram submetidos à avaliação cognitiva e funcional.

\subsection{Avaliação cognitiva}

\subsubsection{Análise intergrupos}

O grupo NC teve desempenho significativamente inferior em relação ao grupo CS na avaliação cognitiva. Os testes mais alterados incluíram funções executivas (EDD, EDI, TTA, TTB e TICS), memória verbal e não verbal (MV, BBRC), praxia construtiva (PC) e fluência verbal semântica (FVS).

Pacientes do grupo NC também tiveram desempenho significativamente pior que os do grupo EC, em testes que avaliaram memória operacional (EDD, EDI), funções executivas (TTB, TICS), linguagem (TN, FVS), praxia construtiva (PC) e orientação visual-espacial (DR) (TABELA 2, FIGURA 1). A frequência de crises epilépticas e o número de DAE utilizadas não se correlacionaram com nenhum dos escores cognitivos dos pacientes do grupo $\mathrm{NC}(\mathrm{p}>0,10$; teste de correlação de Spearman).

O grupo EC apresentou escores inferiores ao grupo CS em testes de funções executivas (EDD, EDI), orientação visual-espacial (TR), memória (evocação tardia de BBRC e MV) e praxia construtiva (TABELA 3). 


\subsubsection{Análise intragrupo}

Todos os pacientes do grupo NC apresentaram ao menos um escore cognitivo significativamente alterado em relação ao grupo CS. Observou-se média de $13,6 \pm 5,81$ resultados de testes (ou subtestes) cognitivos alterados dentre os 30 que faziam parte da avaliação para cada indivíduo com NC. Mais da metade dos pacientes apresentou escores alterados em domínios como memória (BBRC, MV), atenção (EDD), funções executivas (EDI, TICS), linguagem (TN, FVS) e praxia construtiva (PC) (TABELA 4).

O diagnóstico de demência foi preenchido por 5 (12,5\%) pacientes com NC. Pacientes com e sem demência não diferiram significativamente em relação à idade $(44,4 \pm 10,0$ e 38,5 $\pm 5,4$ anos; $\mathrm{p}=0,26)$, nível educacional $(4,2 \pm 3,9$ e $6,2 \pm 3,6$ anos; $p=0,23)$, presença de tratamento prévio para depressão com sucesso $(20,0 \%$ e $28,0 \% ; p=0,97)$, tipo e número de DAE $(1,0 \pm 0,0$ e $1,0 \pm$ $0,2 ; \mathrm{p}=0,64)$, duração da doença $(8,6 \pm 7,0$ e 7,1 $\pm 6,0$ anos; $\mathrm{p}=0,52)$ e frequência mensal de crises epilépticas $(6,2 \pm 1,7$ e 5,3 $\pm 2,5 ; p=0,58)$. Como grupo, pacientes com NC sem demência apresentaram resultados significativamente alterados quando comparados com os grupos EC e CS em funções executivas (EDD, EDI, TTA, TTB), linguagem (FVS) e memória (BBRC e MV) (TABELA 5).

O CCSD foi diagnosticado em 18 pacientes do grupo NC (45\%) (TABELA 4). Pacientes do grupo $\mathrm{NC}$ com e sem CCSD não diferiram significativamente 
quanto à idade $(41,1 \pm 11,9$ e $37,7 \pm 9,1$, respectivamente; $\mathrm{p}=0,39)$ e escolaridade $(5,0 \pm 3,2$ e 7,1 $\pm 4,4$ anos, respectivamente; $p=0,18)$.

O número médio de testes cognitivos alterados por paciente não foi diferente entre o grupo de pacientes com $\mathrm{NC}$ que originalmente se apresentaram com depressão e aqueles que não apresentavam essa síndrome no momento da inclusão no estudo $(7,8 \pm 2,1$ e $6,6 \pm 2,4$, respectivamente; $p=0,97)$.

\subsection{Análise de neuroimagem}

Não se encontrou correlação significativa entre os escores dos testes cognitivos e número, fase evolutiva das lesões ou sua localização (tanto global quanto dividida por lobos cerebrais) à RM. Pacientes com NC com e sem demência não se diferenciaram quanto ao número total de lesões $(12,6 \pm 7,2$ e 19,6 \pm 24,7 , respectivamente; $p=0,86$ ). A presença da forma racemosa também foi semelhante em ambos os grupos $(20,0 \%$ e $17,0 \%$, respectivamente; $p=0,99)$.

\subsection{Poder estatístico do estudo}

Tomando os resultados do MEEM como modelo de teste de avaliação global, o cálculo do poder estatístico da comparação entre os grupos $\mathrm{NC}$ e EC foi o seguinte:

Diferença entre a média mais alta $(\mathrm{EC}: \mathrm{n}=28 ; 27,71 \pm 1,72)$ e mais baixa $(\mathrm{NC}$ : $\mathrm{n}=40 ; 25,43 \pm 3,03)$, assumindo-se que ambos os grupos são independentes, temos que a probabilidade de erro do tipo I associada a esse teste na hipótese 
nula é de 0,004. A proporção de participantes entre os dois grupos é 40:28 $(1,429)$, logo, o poder da comparação é de 0,990 . 


\section{DISCUSSÃO}

No presente estudo foi avaliado o perfil cognitivo de pacientes com $\mathrm{NC}$ sem tratamento específico. Todos os participantes tinham ao menos uma lesão cística com escólex ao exame de RM de crânio, apresentando assim NC ativa e definitiva por critérios absolutos, de acordo com os critérios diagnósticos atuais (Del Brutto et al, 2001).

Este é o primeiro estudo controlado a avaliar o perfil cognitivo de pacientes com NC. Para tal, foram examinados dois grupos controle cuidadosamente emparelhados aos pacientes com NC: o primeiro formado por indivíduos saudáveis, e o segundo por pacientes com epilepsia criptogênica medicados com DAE.

Os pacientes com NC apresentaram declínio cognitivo significativo quando comparados aos CS. Devido à possibilidade de etiologia multifatorial do declínio cognitivo na $\mathrm{NC}$, foram excluídos da avaliação os pacientes apresentando delirium, idade avançada, crises epilépticas recentes, intoxicação por DAE, transtornos infecciosos e hormonais, bem como sintomas depressivos persistentes. Declínio cognitivo foi identificado em quase dois terços dos casos de $\mathrm{NC}$, sendo que 12,5\% (5 em 40) preencheram critérios diagnósticos para demência. Os domínios cognitivos mais frequentemente acometidos foram funções executivas, memória verbal, habilidades visuaisespaciais e linguagem.

Nossos achados salientam a alta prevalência de declínio cognitivo na NC ativa, que o coloca como uma das manifestações clínicas mais frequentes dessa doença. Sua presença é habitualmente subestimada tanto nas séries de casos 
relatadas previamente quanto na prática clínica, provavelmente porque os diferentes domínios cognitivos foram raramente avaliados de forma estruturada (Tretiakoff et al, 1924; Canelas, 1962; Forlenza et al, 1997).

Pacientes com NC apresentam um continuum crescente de declínio cognitivo, iniciando-se com $100 \%$ dos pacientes que apresentam resultados alterados em ao menos um subteste de nossabateria, passando pelo perfil amnéstico sem declínio funcional, presente em $45 \%$ dos doentes (CCSD), e chegando ao extremo de demência definida, observada em 12,5\% deste grupo.

Depressão é uma comorbidade frequente e relevante em NC, que pode afetar o funcionamento cognitivo. Alguns autores (Forlenza et al, 1997) encontraram uma alta prevalência de depressão em pacientes com $\mathrm{NC}(52,6 \%)$, ao passo que outros sequer a avaliaram (Ramirez-Bermudez et al, 2005). Em ambos os casos, os resultados da avaliação cognitiva não foram controlados segundo a presença ou não de depressão tornando-se impossível a distinção de declínio cognitivo relacionado à síndrome depressiva daquele secundário à NC.

No presente estudo, a depressão estava presente em $27,5 \%$ dos pacientes com a forma ativa de NC. Devido a essa alta prevalência, optamos por não avaliar inicialmente os indivíduos deprimidos. Ao invés disso, prescrevemos tratamento farmacológico e a avaliação cognitiva foi realizada somente após um adequado controle sintomático da síndrome depressiva. Tal estratégia mostrou-se eficaz, uma vez que somente um paciente dentre 11 deprimidos deixou de ser avaliado. É importante salientar que quando avaliamos essas duas subpopulações do grupo - NC - os pacientes que se apresentaram inicialmente deprimidos e que foram tratados de forma satisfatória, e aqueles 
que inicialmente não estavam deprimidos - não encontramos diferença estatisticamente significativa entre seus escores nos testes cognitivos. Da mesma forma, a prevalência de antecedente de depressão nestes dois subgrupos não foi diferente do ponto de vista estatístico, o que sugere que a depressão encontrada nos doentes com NC fosse relativamente recente, estando relacionada à $\mathrm{NC}$ e não devido à simples prevalência esperada na população geral.

A comparação dos perfis cognitivos entre os grupos NC e CS é essencial para se determinar a presença de declínio cognitivo e demência nesses grupos de pacientes. No entanto, esta abordagem não fornece informações sobre os mecanismos implicados nesse declínio, uma vez que o desempenho cognitivo também pode ser influenciado por variáveis presentes em indivíduos com NC, como epilepsia e o uso de DAE. Assim, um segundo grupo controle apresentando frequências similares destas mesmas variáveis nos permitiu explorar esta questão.

Pacientes com EC serviram bem a este propósito, uma vez que apresentam crises epilépticas, fazem uso crônico de DAE e não possuem lesões identificáveis no encéfalo que poderiam interferir na avaliação cognitiva. Além disso, este grupo de pacientes não apresenta alterações cognitivas basais tão significativas (Huang et al, 2005).

Pacientes com NC apresentaram um desempenho significativamente inferior ao grupo de pacientes com EC em diversos domínios cognitivos, como funções executivas, fluência verbal, memória episódica verbal e habilidades visuaisespaciais, independentemente da idade, nível educacional, frequência de 
epilepsia e do uso de DAE. Uma vez que indivíduos com EC não apresentam lesões cerebrais detectáveis, as diferenças encontradas são provavelmente relacionadas às lesões da $\mathrm{NC}$ ou a suas complicações diretas como edema cerebral e inflamação local. O declínio cognitivo da NC não pode ainda ser creditado a vieses relacionados a delirium, crises convulsivas, idade avançada, baixo nível educacional ou transtornos do humor.

É interessante notar que os escores cognitivos não se correlacionaram aos achados de neuroimagem à RM, no que diz respeito aos tipos, localização e número de lesões relacionadas à NC. Um estudo anterior também encontrou alta prevalência de declínio cognitivo global em pacientes com $\mathrm{NC}(87,5 \%)$, mas similarmente não encontrou correlação entre estes achados e dados de exames de neuroimagem (Forlenza et al, 1997). O declínio cognitivo da NC não parece ser influenciado pela carga de doença determinada somente pelo número de lesões, mas possivelmente pela interação sinérgica entre número, localização, diferentes fases evolutivas, produção local de inflamação e edema cerebral (Góngora-Rivera et al, 2008). No entanto, cabe ressaltar que somente a avaliação de uma amostra maior de pacientes permitiria confirmar a ausência da correlação entre cognição e imagem de forma definitiva (RamirezBermudez et al, 2005).

De acordo com o DSM-IV, a demência esteve presente em 12,5\% dos pacientes do grupo NC. Pacientes com e sem demência apresentaram idade, nível educacional, frequência de depressão, número médio e tipo de DAE, frequência de crises epilépticas e duração de doença semelhantes. Tais dados sugerem que a demência está primariamente relacionada à $\mathrm{NC}$ e não a outros fatores (Ramirez-Bermudez et al,2005). 
Até hoje, somente um estudo investigou a presença de demência na NC (Ramirez-Bermudez et al, 2005 e avaliou 82 pacientes com NC que apresentavam tanto a forma ativa como inativa da doença. A demência foi detectada em $17 \%$ dos pacientes. Alguns fatores podem explicar a diferença encontrada em relação ao nosso estudo. A presença de depressão no estudo citado não foi avaliada, o que afeta uma parcela significativa de pacientes com NC e pode apresentar impacto negativo sobre o desempenho cognitivo (Bertelson et al, 2003). Além disso, apesar do diagnóstico de demência ter sido baseado nos critérios do DSM-IV, os escores cognitivos não foram fornecidos na publicação.

A inclusão de indivíduos é outro potencial fator de confusão a se levar em conta, uma vez que outras causas de declínio cognitivo passam a ser mais frequentes nessa população, fazendo com que demências de outras etiologias sejam corretamente diagnosticadas mas erroneamente creditadas à NC como fator causal. Efetivamente, o grupo de pacientes dementes neste estudo (Ramirez-Hernandez et al, 2005) apresentava médias de idade e de nível de escolaridade muito diferentes daqueles sem demência, o que corrobora esta hipótese.

Nosso estudo proporciona uma visão transversal do perfil cognitivo dos pacientes com $\mathrm{NC}$ ativa. $\mathrm{O}$ seguimento destes pacientes é uma tarefa de difícil realização pelo fato de que a maior parte dos pacientes apresentava múltiplas lesões, muitas em fases diferentes de evolução, indo de cistos intactos a cistos calcificados, passando por todo o contínuo da degeneração (formas transicionais). Cada uma dessas fases apresenta maior ou menor predisposição à formação de edema, inflamação local, propensão a delirium, crises 
epilépticas e ainda outras condições associadas (como efeitos colaterais de DAE e depressão). Isso leva a apresentações clínicas altamente heterogêneas, podendo variar desde a ausência total de sintomas até a presença de status epilepticus. A este contexto, podemos adicionar os efeitos temporalmente dinâmicos das medicações, crises epilépticas e transtornos do humor. Assim, em um indivíduo isolado, a NC tem um perfil dinâmico. Avaliações pontuais podem tanto superestimar quanto subestimar a prevalência de CCSD e demência, dependendo da presença de todos estes fatores. Por outro lado, avaliações longitudinais de pacientes com NC são sujeitas a muitos vieses e limitações (Ramirez-Bermudez et al, 2005).

Por outro lado, não resta dúvida que o seguimento dos pacientes com CCSD e demência permitiria uma melhor compreensão da história natural do declínio cognitivo associado à NC. Pode-se questionar se o quadro cognitivo não melhoraria com a resolução espontânea ou em consequência ao tratamento farmacológico dos cistos ativos, assim como ocorre com outros sintomas clínicos da $\mathrm{NC}$ como as crises convulsivas e os déficits neurológicos focais. Poder-se-ia ainda questionar se o declínio cognitivo não se comportaria como a síndrome de $\mathrm{HIC}$, que precisa ser abordada e tratada rapidamente para que danos irreversíveis sejam prevenidos.

Estudos controlados são necessários para responder essa questão e para avançar nosso conhecimento acerca dos mecanismos fisiopatológicos da NC. De qualquer modo, com base nos achados do presente estudo é possível dizer que o declínio cognitivo parece ser uma das apresentações clínicas mais frequentes da $\mathrm{NC}$ ativa e que, portanto, deve entrar de forma sistemática no rol 
da avaliação clínica dessa doença, tanto em protocolos de pesquisa quanto na prática clínica.

\section{CONCLUSÕES}

1. No presente estudo, a $\mathrm{NC}$ em sua fase ativa associou-se de forma significativa a comprometimento cognitivo. Todos os pacientes avaliados apresentaram escores em testes cognitivos abaixo dos valores normais quando comparados a CS. Quase metade dos pacientes apresentou CCSD, e 12,5\% apresentaram demência.

2. Os domínios cognitivos mais acometidos nos pacientes com $\mathrm{NC}$ ativa em comparação a CS foram funções executivas, memória verbal e não verbal, praxia construtiva e fluência verbal. Quando comparado ao grupo EC, o grupo NC apresentou comprometimento significativo em memória operacional, memória episódica verbal, funções executivas, nomeação, praxia construtiva e habilidades visuais-espaciais.

3. Pacientes com NC apresentaram comprometimento cognitivo mesmo quando comparados a indivíduos com EC com frequência de crises epilépticas e uso de DAE semelhantes.

4. O comprometimento cognitivo dos pacientes com $\mathrm{NC}$ ativa não mostrou associação a achados de neuroimagem por RM. 


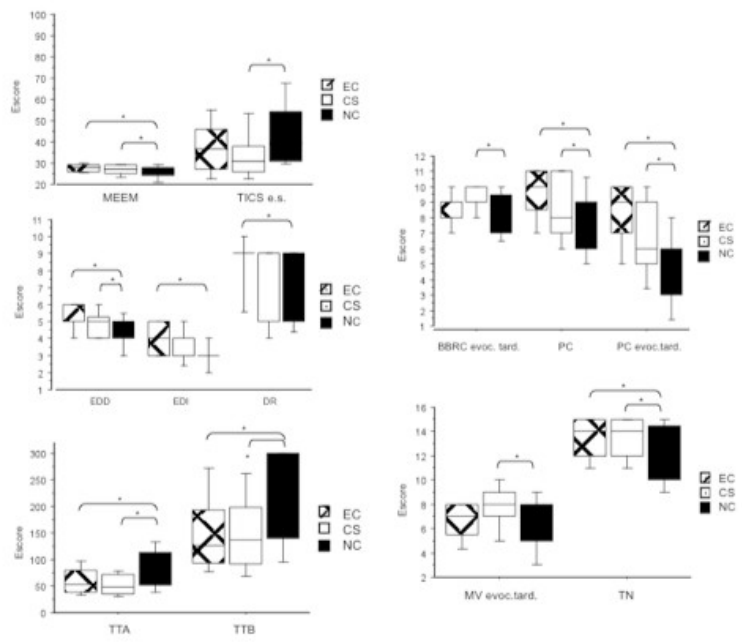

Figura 1 
Tabela 1: Distribuição da idade, anos de educação e uso de drogas antiepilépticas e frequência de crises epilépticas entre os grupos.

\begin{tabular}{|c|c|c|c|c|c|c|c|c|c|c|c|c|c|}
\hline & \multicolumn{4}{|c|}{ idade } & \multicolumn{5}{|c|}{ anos de educação } & \multirow[t]{2}{*}{ CMZ } & \multirow[t]{2}{*}{ PHT } & \multirow[t]{2}{*}{ PB } & \multirow[t]{2}{*}{$\begin{array}{l}\text { crises / } \\
\text { mês. }\end{array}$} \\
\hline & $18-30$ & $31-40$ & $41-50$ & 51-60 & analfabeto & 1 a 4 & 5 a 8 & 9 a 12 & $>12$ & & & & \\
\hline $\mathrm{NC}$ & $11(25 \%)$ & $12(30 \%)$ & $10(25 \%)$ & $7(17 \%)$ & $2(5 \%)$ & $16(40 \%)$ & $10(25 \%)$ & $10(25 \%)$ & $2(5 \%)$ & $62,50 \%$ & $25,80 \%$ & $25,80 \%$ & $5,4 \pm 2,3$ \\
\hline Cs & $13(26 \%)$ & $13(26 \%)$ & $12(24 \%)$ & $11(22 \%)$ & $1(2 \%)$ & $16(32 \%)$ & $13(26 \%)$ & $18(36 \%)$ & $1(2 \%)$ & . & - & - & - \\
\hline EC & $9(32 \%)$ & $5(17 \%)$ & $8(28 \%)$ & $6(21 \%)$ & $1(3 \%)$ & $7(25 \%)$ & $9(32 \%)$ & $10(35 \%)$ & $1(3 \%)$ & $65,50 \%$ & $17,20 \%$ & $17,20 \%$ & $4,8 \pm 1,7$ \\
\hline
\end{tabular}

Tabela 2: Resultados do testes cognitivos e funcionais dos grupos NC, EC e CS

\begin{tabular}{|c|c|c|c|c|c|c|c|c|c|c|c|c|c|c|c|c|}
\hline \multicolumn{2}{|c|}{$\begin{array}{c}\text { TESTE } \\
\text { COGNITVO }\end{array}$} & NEEM & 500 & EOI & $\pi \cdot A$ & 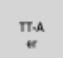 & $\pi \cdot 8$ & $\pi \cdot B$ ar & $\mathrm{TN}$ & $\begin{array}{c}\text { B8RC. } \\
\text { perc. }\end{array}$ & $\begin{array}{c}\text { Berc. } \\
\text { nom }\end{array}$ & BBAC- & $\begin{array}{l}\text { B8BC } \\
\text { imed.1 }\end{array}$ & ВвяС. & $\begin{array}{c}\text { BBRC. } \\
\text { evose. tard }\end{array}$ & BCSTryec. \\
\hline \multirow{3}{*}{ ESCORE } & Nc & $\begin{array}{c}25,43 \pm \\
3,03 t\end{array}$ & $\begin{array}{c}4.45 \pm 1,1 \\
1 \pi\end{array}$ & $\begin{array}{c}3,05 \pm 0,88 \\
\pi\end{array}$ & $\begin{array}{c}84,54 \pm 42,7 \\
\text { of }\end{array}$ & $0.31 \pm 0,66$ & $\begin{array}{c}214,78 \pm \theta 3,0 \\
2 \pi\end{array}$ & $1,3111,60$ & $\begin{array}{c}11,90 \pm 2,2 \\
6 \pi\end{array}$ & $10,00 \pm 0.0$ & $\begin{array}{l}9,99 \pm 0,1 \\
6\end{array}$ & $6.55 \pm 1.22$ & $\begin{array}{c}8,30 \pm 1,30 \\
\pi\end{array}$ & $\begin{array}{l}8.93+1.27 \\
\pi\end{array}$ & $8,30 \pm 1,57+1$ & $9,8000,61+$ \\
\hline & cs & $\frac{26,76 \pm 2.3}{3 *}$ & $\begin{array}{c}4.84+0.9 \\
6^{\circ}\end{array}$ & $3,3390.92$ & $\begin{array}{c}54.96 \pm 24,3 \\
1=\end{array}$ & $0.35 \pm 0.56$ & $\begin{array}{c}149,86 \pm 71,1 \\
4^{*}\end{array}$ & $0,83 \pm 1,40$ & $\begin{array}{c}13,10 \pm 2,0 \\
g^{*}\end{array}$ & $10,0000.0 .0$ & $\begin{array}{l}9,96 \pm 0,1 \\
4\end{array}$ & $6.96+1.26$ & $9,14 \pm 0,89$ & $9.61 \pm 0.61$ & $9,2440,85^{* *}$ & $10,00 \pm 0,00$ \\
\hline & EC & $\begin{array}{c}27,7111,7 \\
211 \mathrm{w}\end{array}$ & $\begin{array}{c}5.9+0.8 \\
5 \mathrm{~min}\end{array}$ & $\begin{array}{c}3.9640 .96 \\
1 \text { w }\end{array}$ & $\begin{array}{c}59.25 \pm 28,1 \\
6\end{array}$ & $0.21 \pm 0,50$ & $\begin{array}{c}14,61 \pm 71,7 \mathrm{~d} \\
\text { " }\end{array}$ & $0,86 t 1,15$ & $\begin{array}{c}13,57 \pm 1,7 \\
{ }_{51 \mathrm{II}}\end{array}$ & $10,00 \pm 0.0$ & $\begin{array}{c}10,00 \pm 0, \\
\infty\end{array}$ & $6.50 \pm 1.04$ & $8,71 \pm 1,91$ & $2.14 \pm 0,71$ & $8.50 \pm 1.07$ & $10,00 \pm 0,00$ \\
\hline \multicolumn{2}{|c|}{$\begin{array}{l}\text { TESTE } \\
\text { COGNITIV }\end{array}$} & $\begin{array}{l}\text { BBRC } \\
\text { ints. }\end{array}$ & TR & Fvs & MV-imed. & $\begin{array}{c}\text { MV-svoc. } \\
\text { tar. }\end{array}$ & WV-roe. & Tics.e. & $\begin{array}{c}\text { nces-es. } \\
\text { ar. }\end{array}$ & TiCS.L. & $\begin{array}{l}\text { Tics - } \\
\text { Lear. }\end{array}$ & rics-s.e. & $\begin{array}{l}\text { Tres- } \\
\text { e.s.ert. }\end{array}$ & PC & $\begin{array}{c}\text { PC.evoc } \\
\text { tard. }\end{array}$ & PFAQ \\
\hline \multirow{3}{*}{ ESCORE } & NC & $0,58 \pm 2224$ & $\frac{7.31 \pm 2,3}{6+}$ & $\begin{array}{c}13,77 \pm 4.2 \\
9 \% 4\end{array}$ & $\underset{\pi}{18.25+3.98}$ & 6,18ะ2, 18 & $18,5371,834$ & $\begin{array}{c}24,47 \pm 102 \\
31+1\end{array}$ & $0.34 \pm 1.07$ & $\begin{array}{c}23.06415 .88 \\
811\end{array}$ & $\frac{0.20 \pm 0,7}{6}$ & $\frac{4,200416,4}{4}$ & $\underset{2.11 \pm 2.693}{\pi}$ & $\begin{array}{c}211 \pm 2.53 \\
+1+1\end{array}$ & $4,852,2,65+1$ & $1.58+3.7,711$ \\
\hline & cs & $0,00 \pm 0.000$ & $7,77 \pm 2.2$ & $\begin{array}{l}17,65 \pm 5,4 \\
0^{*-5}\end{array}$ & 21.2993 .320 & 79001,70 & $19,69 \pm 0.55^{*}$ & $17,14 \pm 6.00^{\circ}$ & $0,00 \pm 0.00$ & $15,10 \pm 5,80$ & $0,14 \pm 0,6$ & $5, \frac{22213,7}{7 * 7}$ & $0,7641,36$ & $0.76 \pm 1.36$ & $6,67 \pm 2,49^{-}$ & $00,00 \pm 0,00$ \\
\hline & EC & $0,71 \pm 262$ & $\begin{array}{c}8.8641,6 \\
6: 5\end{array}$ & $16,43 \pm 4,5$ & $20,29+3,41$ & $6.68 \pm 185$ & $19.35+0,83$ & $18,3244,731$ & 0,0000000 & $\begin{array}{c}14.50 \pm 4.87 \\
11.87\end{array}$ & $\frac{0,07 \pm 0,3}{8}$ & $\frac{9,07713,8}{2}$ & $\frac{0.84+1,19}{n}$ & $\begin{array}{l}0.64 \pm 1,19 \\
\text { win }\end{array}$ & $8,25 \pm 1,645$ & $0,07 \pm 0.38$ \\
\hline
\end{tabular}

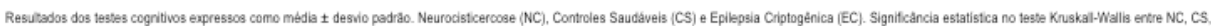

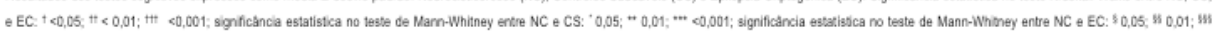

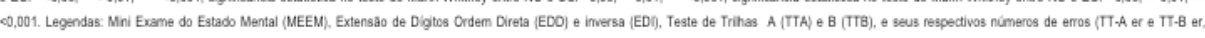

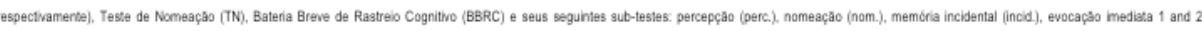

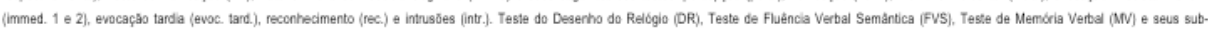

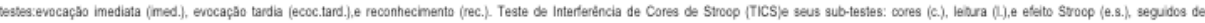

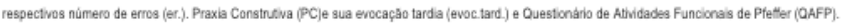


Tabela 3: Comparação do desempenho cognitivo e funcional entre os grupos controle.

\begin{tabular}{|c|c|c|c|c|c|c|c|c|c|c|c|c|c|c|c|}
\hline $\begin{array}{c}\text { TESTE } \\
\text { COGNITIVO }\end{array}$ & MEEM & 500 & EOI & $\pi \cdot A$ & $\stackrel{\pi \cdot A}{*}$ & $\pi \cdot 8$ & $\pi \cdot B$ or & $\pi \mathbb{N}$ & $\begin{array}{c}\text { serc. } \\
\text { pere. }\end{array}$ & $\begin{array}{c}\text { B8pc. } \\
\text { nom }\end{array}$ & $\begin{array}{l}\text { BBRC. } \\
\text { incid }\end{array}$ & $\begin{array}{l}\text { Barc } \\
\text { imed.1 }\end{array}$ & $\begin{array}{l}\text { BBRC. } \\
\text { imed.2 }\end{array}$ & $\begin{array}{c}\text { BERC. } \\
\text { eves. tard }\end{array}$ & BCST-нс. \\
\hline cs & $\begin{array}{c}26,76 \pm 2,3 \\
3\end{array}$ & $\begin{array}{c}4.84 \pm 0,9 \\
6\end{array}$ & $3,33 \pm 0.92$ & $54,96 \pm 24,1$ & 0 & $149,96 \pm 71,4$ & $0,80 \pm 1,40$ & $13,10 \pm 2,9$ & $10,00 \pm 0,0$ & $9,9840,1$ & $6.9651,26$ & 9.1400 .89 & 9.6110.51 & $9.24 \pm 0.85$ & $10,00 \pm 0,00$ \\
\hline EC & $\begin{array}{c}27,71 \pm 1,7 \\
2\end{array}$ & $\begin{array}{c}529+0.8 \\
55\end{array}$ & $\begin{array}{c}3.9650 .95 \\
7.06\end{array}$ & $\begin{array}{c}50,25+26,1 \\
6\end{array}$ & $0.21 \pm 0,50$ & $\begin{array}{c}147,61+71.7 \\
8\end{array}$ & $0,86 \pm 1,15$ & $\begin{array}{c}13,57 \pm 1,7 \\
5\end{array}$ & $10,00 \pm 0,0$ & $\begin{array}{c}10,000 \pm 0, \\
\infty\end{array}$ & $6.5011,04$ & $8.71 \pm 1,91$ & $\frac{2.140 .71}{48}$ & 8,501,07\% & $10,00 \pm 0.00$ \\
\hline $\begin{array}{l}\text { TESTE } \\
\text { COGNITIV }\end{array}$ & $\begin{array}{c}\text { BeRC } \\
\text { int. }\end{array}$ & TR & Fvs & MV-imed. & $\begin{array}{c}\text { WV-evoc. } \\
\text { tar. }\end{array}$ & WW roe & rics.e. & $\begin{array}{l}\text { nics-e. } \\
\text { * }\end{array}$ & Ties.t. & 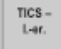 & TICS-s.e. & $\begin{array}{l}\text { Tics- } \\
\text { e.s. }\end{array}$ & PC & $\begin{array}{c}\text { PC.evoce } \\
\text { tard. }\end{array}$ & PFAQ \\
\hline cs & $0,00 \pm 0,00$ & $\frac{7.47 \pm 2.2}{7}$ & 17.6555,4 & $21,29 t 320$ & 79001,70 & $19,69 \pm 0,55$ & $17,14+6,00$ & $0,00 \pm 0.00$ & $15,10 \pm 5,80$ & $0,14 \pm 0,6$ & $35,02+13,7$ & $0,76 \pm 1,36$ & $0.7651,36$ & $6.67 \pm 2.49$ & $00,00 \pm 0,00$ \\
\hline EC & $0,71 \pm 2.62$ & $\frac{8.64+1.6}{45}$ & $\begin{array}{c}16,4344,5 \\
3\end{array}$ & $20,29+3,41$ & $\frac{6.68+1,15}{11}$ & 19,3600,835 & $\underset{18,324}{1}$ & $0,00 \pm 0.000$ & $14,5004,87$ & $0,07 \pm 0,3$ & $\frac{5,07 \pm 13,3}{2}$ & $0.8441,19$ & $\frac{0.64 t 1,19}{8}$ & $825+1,845$ & $02,07 \pm 0,38$ \\
\hline
\end{tabular}

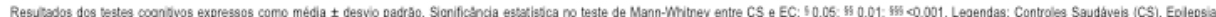

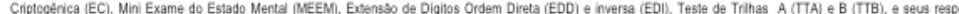
(c) (1)

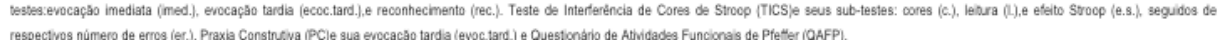

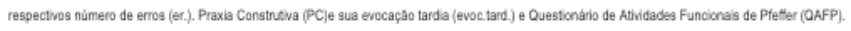

Tabela 4

\begin{tabular}{|c|c|c|c|c|c|c|c|c|c|c|c|c|c|c|c|}
\hline $\begin{array}{l}\text { TESTE } \\
\text { COGNI } \\
\text { TIVO }\end{array}$ & MEEM & EDD & EDI & $\pi-A$ & $\begin{array}{l}\text { TT-A } \\
\text { er }\end{array}$ & $T \pi-B$ & $\underset{\text { er }}{T-B}$ & TN & $\begin{array}{c}\text { BBRC- } \\
\text { perc. }\end{array}$ & $\begin{array}{l}\text { BBRC- } \\
\text { nom. }\end{array}$ & $\begin{array}{l}\text { BBRC- } \\
\text { inoid. }\end{array}$ & $\begin{array}{l}\text { BBRC } \\
\text { imed.1 }\end{array}$ & $\begin{array}{l}\text { BBRC- } \\
\text { imed. } 2\end{array}$ & $\begin{array}{l}\text { BBRC- } \\
\text { evoo. } \\
\text { tard }\end{array}$ & $\begin{array}{c}\text { BCsT- } \\
\text { rec. }\end{array}$ \\
\hline $\begin{array}{c}\text { Eseore } \\
\text { médio } \\
\text { CS }: \\
\text { d.p.lex } \\
\text { tensío } \\
\text { I: IC }\end{array}$ & $\begin{array}{c}26,7542 \\
33(22- \\
30), \\
0,65\end{array}$ & $\begin{array}{c}4,83 * 0, \\
96(3-7) ; \\
0,27\end{array}$ & $\begin{array}{c}3,3220, \\
92(2-9) ; \\
0,26\end{array}$ & $\begin{array}{c}54,95 * 2 \\
4,30(5- \\
135) ; \\
6,81\end{array}$ & $\begin{array}{c}0,34+0, \\
6 \sigma(0-3) ; \\
0,19\end{array}$ & $\begin{array}{c}149,954 \\
71,13(5 \\
5.300) ; \\
19,92\end{array}$ & $\begin{array}{c}0,83 * 1, \\
40(0-3) ; \\
0,39\end{array}$ & $\begin{array}{c}13,10 \div 2 \\
, 08(5- \\
15) ; \\
0,58\end{array}$ & $10 \pm 0,0$ & $\begin{array}{c}9,98+0, \\
14(9- \\
10) ; \\
0,04\end{array}$ & $\begin{array}{c}6,85 * 1, \\
25(4- \\
10) ; \\
0,35\end{array}$ & $\begin{array}{l}9,14 \pm 0, \\
897- \\
107 ; \\
0,25\end{array}$ & $\begin{array}{c}9,600,6 \\
0(8,10) ; \\
0,17\end{array}$ & $\begin{array}{l}9,24 \pm 0, \\
85(7- \\
10 ; \\
0,24 \\
0,24\end{array}$ & $10 \pm 0,0$ \\
\hline $\begin{array}{c}\text { Númer } \\
\text { o de } \\
\text { pacien } \\
\text { tes } \\
\text { com } \\
\text { NC } \\
\text { com } \\
\text { scores } \\
\text { alterad } \\
\text { os (\$6) }\end{array}$ & $\begin{array}{c}24 \\
(6006)\end{array}$ & $\begin{array}{c}21 \\
(52,5 \% 6)\end{array}$ & $\begin{array}{c}32 \\
(8005)\end{array}$ & $\begin{array}{c}25 \\
(62,5 \%)\end{array}$ & $8(2096)$ & $\begin{array}{l}24 \\
(60 \% 6)\end{array}$ & $\begin{array}{c}12 \\
(30 \%)\end{array}$ & $\begin{array}{c}23 \\
(57,5 \% 6)\end{array}$ & 0 & $\stackrel{1}{1}$ & $\begin{array}{c}19(47,5 \\
96)\end{array}$ & $\begin{array}{l}19(47,5 \\
\text { (6) }\end{array}$ & $\begin{array}{c}23 \\
(57,5 \% 6)\end{array}$ & $\begin{array}{c}30 \\
(75 \%)\end{array}$ & 0 \\
\hline $\begin{array}{l}\text { TESTE } \\
\text { COGNN } \\
\text { TIVO }\end{array}$ & $\begin{array}{c}\text { BBRC } \\
\text { intr. }\end{array}$ & TR & FVS & $\begin{array}{l}\text { Mv- } \\
\text { imed. }\end{array}$ & $\begin{array}{c}\text { MV- } \\
\text { evoc. } \\
\text { tar. }\end{array}$ & $\begin{array}{l}\text { Mv- } \\
\text { rec. }\end{array}$ & TICS-c. & $\begin{array}{l}\text { Tics- } \\
\text { c.er. }\end{array}$ & TICS-1. & $\begin{array}{c}\text { Tics - } \\
\text { Ler. }\end{array}$ & $\begin{array}{l}\text { Tics - } \\
\text { s.e. }\end{array}$ & $\begin{array}{l}\text { nics - } \\
\text { e.s.er. }\end{array}$ & PC & $\begin{array}{l}\text { PC. } \\
\text { evoc } \\
\text { tard. }\end{array}$ & PFAQ \\
\hline 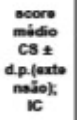 & $0.0 \pm 0.0$ & $\begin{array}{c}7,46 \pm 2 \\
27(2- \\
10) ; \\
0.04\end{array}$ & $\begin{array}{c}17.65 \pm \\
5.39(8- \\
31) ; \\
1.51\end{array}$ & $\begin{array}{c}21.28 \pm \\
3.19(13 \\
-277 ; \\
0.89\end{array}$ & $\begin{array}{c}7.80 \pm 1 . \\
69(4) \\
10): \\
0.48\end{array}$ & $\begin{array}{c}19,60 \pm \\
0.54(18 \\
-20) \\
0.15\end{array}$ & $\begin{array}{c}17,14 \pm \\
0.00(10 \\
-35) ; \\
1.08\end{array}$ & $0.0 \pm 0.0$ & \begin{tabular}{|c}
$15,10 \pm$ \\
$5.79(7-$ \\
$39) ;$ \\
1.02 \\
\end{tabular} & $\begin{array}{c}0.14 \pm 0 . \\
64(0- \\
4): 0.18\end{array}$ & $\begin{array}{c}35.024 \\
13.77(1 \\
8.76): \\
3.08\end{array}$ & $\begin{array}{r}0,75 \pm 1 . \\
36(0- \\
6) ; 0.38\end{array}$ & $\begin{array}{c}\text { 8,56:1. } \\
92(5- \\
11) ; \\
0.54\end{array}$ & $\begin{array}{c}6,67 \pm 2 . \\
48(1- \\
10) \cdot \\
0.70\end{array}$ & $\begin{array}{c}10.0 \pm 0 \\
0 ;\end{array}$ \\
\hline 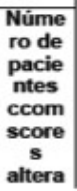 & 0 & $\begin{array}{c}13 \\
(32,55)\end{array}$ & $(77.5 \%)$ & $\begin{array}{c}27(67, \\
5 \%)\end{array}$ & 28 (700) & $\begin{array}{c}23 \\
(57.5 \mathrm{x})\end{array}$ & $24(5005)$ & $\begin{array}{c}6 \\
(15 \%)\end{array}$ & $\begin{array}{c}21 \\
(52,5 \\
\%)\end{array}$ & $\begin{array}{c}4 \\
(10 \%)\end{array}$ & $\begin{array}{c}18 \\
(45 \%)\end{array}$ & $\begin{array}{c}17 \\
(42,5 \\
\%)\end{array}$ & $\begin{array}{c}27 \\
(67.5 \mathrm{x})\end{array}$ & $\begin{array}{c}23 \\
\text { (57.55) }\end{array}$ & $\begin{array}{c}5 \\
(12,5 \\
\%)\end{array}$ \\
\hline
\end{tabular}


Tabela 4: Resultados dos testes cognitivos e funcionais de indivíduos do grupo Controle Saudável (CS) e número de pacientes do grupo NC com escores alterados.

Legenda. Controles Saudáveis (CS), Neurocisticercose (NC), desvio padrão (d.p.), intervalo de confiança (IC). Mini Exame do Estado Mental (MEEM), Extensão de Dígitos Ordem Direta (EDD) e inversa (EDI), Teste de Trilhas A (TTA) e B (TTB), e seus respectivos números de erros (TT-A er e TT-B er, respectivamente), Teste de Nomeação (TN), Bateria Breve de Rastreio Cognitivo (BBRC) e seus seguintes sub-testes: percepção (perc.), nomeação (nom.), memória incidental (incid.), evocação imediata 1 and 2 (immed. 1 e 2), evocação tardia (evoc. tard.), reconhecimento (rec.) e intrusões (intr.). Teste do Desenho do Relógio (DR), Teste de Fluência Verbal Semântica (FVS), Teste de Memória Verbal (MV) e seus sub-testes:evocação imediata (imed.), evocação tardia (ecoc.tard.),e reconhecimento (rec.). Teste de Interferência de Cores de Stroop (TICS)e seus sub-testes: cores (c.), leitura (I.),e efeito Stroop (e.s.), seguidos de respectivos número de erros (er.). Praxia Construtiva (PC)e sua evocação tardia (evoc.tard.) e Questionário de Atividades Funcionais de Pfeffer (QAFP).

Tabela 5: Resultado de testes cognitivos e avaliação funcional entre pacientes com neurocisticerose não dementes, controles saudáveis e pacientes com epilepsia criptogênica.

\begin{tabular}{|c|c|c|c|c|c|c|c|c|c|c|c|c|c|c|c|c|}
\hline \multicolumn{2}{|c|}{$\begin{array}{c}\text { TESTE } \\
\text { COGNITIVO }\end{array}$} & MEEU & EDO & EDI & $\pi-A$ & $\underset{\text { er }}{\pi-A}$ & $\pi-8$ & $\pi-8$ er & TN & $\begin{array}{l}\text { BBRC. } \\
\text { perc. }\end{array}$ & $\begin{array}{l}\text { B8RC- } \\
\text { nom. }\end{array}$ & $\begin{array}{c}\text { B8RC- } \\
\text { incid. }\end{array}$ & $\begin{array}{l}\text { BBRC } \\
\text { imed.1 }\end{array}$ & $\begin{array}{l}\text { B8RC. } \\
\text { imed. } 2\end{array}$ & $\begin{array}{l}\text { BBRC. } \\
\text { erock tard }\end{array}$ & BCST-tec. \\
\hline \multirow{3}{*}{ EsCORE } & мо & $\begin{array}{l}25.94= \\
271+11\end{array}$ & $\begin{array}{c}4.51 \pm 1.2 \\
11 t\end{array}$ & $\begin{array}{c}\begin{array}{c}3.14 \pm 0.84 \\
m+1\end{array} \\
\end{array}$ & $\begin{array}{c}77.67 \pm 35.7 \\
5 \mathrm{mt}\end{array}$ & $0.29 \pm 0.67$ & $\begin{array}{c}207.53 \pm 84.6 \\
11 \mathrm{II}\end{array}$ & $1.12 \pm 1.36$ & $\begin{array}{c}1200 \pm 22 \\
7 \pi\end{array}$ & 1000000.0 & $\begin{array}{c}997 \pm 0.1 \\
6\end{array}$ & $6.60 \pm 1.26$ & $\begin{array}{c}8.45 \pm 1.24 \\
\pi\end{array}$ & $\begin{array}{c}920 \pm 1.07 \\
\mathrm{tIt}\end{array}$ & $\begin{array}{c}8.51 \pm 1.52 \pi \\
+\end{array}$ & $\frac{9.94 \pm 0.23 t}{+}$ \\
\hline & cs & $\begin{array}{c}26.76+2.3 \\
3\end{array}$ & $\begin{array}{c}4.84 \pm 0.9 \\
6\end{array}$ & $3.33+0.92$ & $\begin{array}{c}54.96+24.3 \\
1 *\end{array}$ & $0.35 \div 0.06$ & $\begin{array}{c}149.96 \pm 71.1 \\
4^{* *}\end{array}$ & $0.83 \div 1.40$ & $\begin{array}{c}13.10=20 \\
9.0\end{array}$ & $10.00 \pm 0.0$ & $\begin{array}{c}9.98 \pm 0.1 \\
4\end{array}$ & $6.86 \pm 1.26$ & $\begin{array}{l}9.14 \pm 0.99 \\
-=\end{array}$ & 9.61:0.61 & $9.24 \pm 0.05^{\circ}$ & $10.00 \pm 0.00$ \\
\hline & EC & $\begin{array}{c}27.71 \pm 1.7 \\
z\end{array}$ & $\begin{array}{c}5.29+0.8 \\
5 \mathrm{~m}\end{array}$ & $\begin{array}{c}3.96 \pm 0.96 \\
=0\end{array}$ & $\begin{array}{c}59.25+26.1 \\
\approx\end{array}$ & 0.2110 .50 & $\begin{array}{c}147.61 \pm 71.7 \\
\mathrm{~mm}\end{array}$ & $0.86 \pm 1.15$ & $\begin{array}{c}13.57 \pm 1.7 \\
5 m\end{array}$ & $10.00 \pm 0.0$ & $\begin{array}{c}1000 \pm 0 \\
\infty\end{array}$ & $6.50 \pm 1.04$ & $8.71 \pm 1.01$ & $9.14 \pm 0.71$ & $8.50 \pm 1.07$ & $10.00 \pm 0.00$ \\
\hline \multicolumn{2}{|c|}{$\begin{array}{c}\text { TESTE } \\
\text { COGNTINO }\end{array}$} & $\begin{array}{l}\text { BBRC } \\
\text { intr. }\end{array}$ & TR & Fvs & wV-imed. & $\begin{array}{c}\text { ulv-evoc. } \\
\text { tar. }\end{array}$ & WV-rece. & nics. & $\begin{array}{c}\text { TICS-c.. } \\
\text { ef. }\end{array}$ & TICs-1. & $\begin{array}{c}\text { Tics- } \\
\text { Let. }\end{array}$ & TiCs-se. & $\begin{array}{l}\text { TCS- } \\
\text { e.s.et. }\end{array}$ & PC & $\begin{array}{c}\text { PC-evoc } \\
\text { tard. }\end{array}$ & PFAQ \\
\hline \multirow{3}{*}{ ESCORE } & мо & $0.57 \pm 2.35$ & $\begin{array}{c}7.61 \pm 20 \\
811\end{array}$ & \begin{tabular}{|c|}
13.744 .2 \\
$5 t 1$
\end{tabular} & $\begin{array}{c}18.743 .3 .79 \\
\pi\end{array}$ & $6.57 \pm 1.97$ & $\underset{\leftarrow}{18.68+1.55 t}$ & $\underset{\pi}{22.8499 .277}$ & $\begin{array}{c}0.33 \pm 1.10 \\
\mathrm{mt}\end{array}$ & $\underset{301}{22000160}$ & $\stackrel{0.1190 .7}{9}$ & 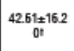 & $\begin{array}{c}1.87 \pm 258 \\
\pi\end{array}$ & $\underset{\substack{211 \geq 2.63 \\
\pi}}{\pi}$ & $\underset{5}{5.02 \pm 2.40+1}$ & $\stackrel{0.37 \pm 0.69 t}{+}$ \\
\hline & cs & $0.00 \pm 0.00$ & $\begin{array}{c}7.77 \pm 2.2 \\
7\end{array}$ & $\underset{17.655 .4 .4}{0.4}$ & $\begin{array}{c}21.29 \pm 3.20 \\
.\end{array}$ & $790 \pm 1.70$ & $19.69 \pm 0.55^{*}$ & $17.1446 .00^{\circ}$ & $0.000 \pm 0.00$ & $15.10 \div 5.80$ & $\begin{array}{c}0.14 \pm 0.6 \\
5\end{array}$ & $\frac{35.02+137}{7^{*}}$ & $0.76 \pm 1.36$ & $0.76 \pm 1.36$ & $6.67 \pm 2.49^{\circ}$ & $0.00 \pm 0.000$ \\
\hline & EC & $0.71 \pm 2.62$ & $\begin{array}{c}8.64 \pm 1.6 \\
4 \mathrm{w}\end{array}$ & \begin{tabular}{|c|}
$16.43 * 4.5$ \\
$3^{\natural}$
\end{tabular} & $20.29+3.41$ & $6.68 \pm 1.85$ & 19.3650 .83 & $18.32 \pm 4.73$ & $\underset{0.00 \pm 0.00}{1}$ & $\underset{4}{14.50+4.87}$ & $\begin{array}{c}0.07 \pm 0.3 \\
8\end{array}$ & $\underset{2}{38.07 \pm 13.3}$ & $\underset{0.64 \pm 1.19}{1.19}$ & \begin{tabular}{|c|c|}
$0.64 \pm 1.19$ \\
wa
\end{tabular} & $\stackrel{8.25 \pm 1.840}{1}$ & $0.07 \approx 0.38$ \\
\hline
\end{tabular}

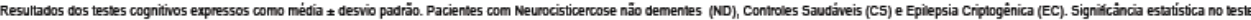

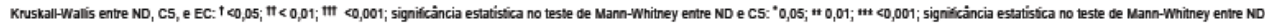

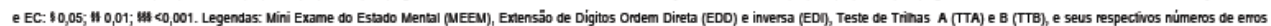

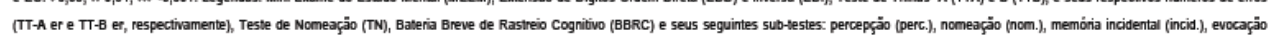

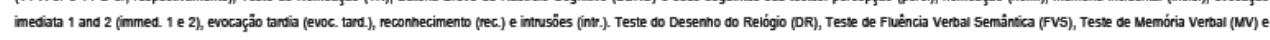

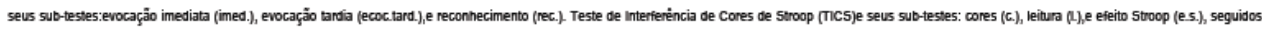

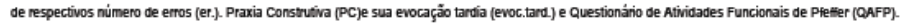




\section{REFERÊNCIAS BIBLIOGRÁFICAS}

Agapejev S. Aspectos Clínicos-Epidemiológicos da Neurocisticercose no Brasil. Arq Neuropsiquiatr. 2003; 61: 822-28.

American Psychiatric Association. Diagnostic and statistical manual of mental disorders, 4th ed. Washington, DC: American Psychiatric Association, 1994. Arriagada C. Clínica de la Neurocisticercosis: I. Manifestaciones Clínicas de la Cisticercosis Encefálica. Neurocirurgia. 1961; 19: 232-47.

Bastos FO. Aspectos psiquiátricos da neurocisticercose encefálica. Rev Paul Med. 1953; 43: 162-64.

Bertelson JA, Price BH. Depression and psychosis in neurological practice. In: Bradley WG, Daroff RB, Fenichel GM, Jankovic J, editors. Neurology in Clinical Practice: Principles of Diagnosis and Management. Boston: Butterworth Heinemann; 2003:103-116.

Bertolucci PH, Brucki SM, Campacci SR, Juliano Y. [The Mini-Mental State Examination in a general population: impact of educational status]. Arq Neuropsiquiatr. 1994; 52: 1-7.

Bertolucci PH, Okamoto IH, Brucki SM, Siviero MO, Toniolo Neto J, Ramos LR. Applicability of the CERAD neuropsychological battery to Brazilian elderly. Arq Neuropsiquiatr 2001; 59: 532-36. 
Bianchin MM, Velasco TR, Takayanagui OM, Sakamoto AC. Neurocysticercosis, mesial temporal lobe epilepsy, and hippocampal sclerosis: an association largely ignored. Lancet Neurol. 2006; 5: 20-1.

Boppré MC, Wille PR, Guarnieri R, Rezek K, Carqueja C, Trevisol-Bittencourt PC, Walz JC, Bianchin M, von Wangenheim A, Chaves ML, Walz R. Cognitive Performance of Patients with Epilepsy and Calcified Neurocysticercotic Lesions: A Case-Control Study. Epilepsy Behav. 2001; 2: 558-62.

Bragazza LM, Vaz AJ, Passos AD, Takayanagui OM, Nakamura PM, Espindola NM, Pardini A, Bueno EC. Frequency of serum anti-cysticercus antibodies in the population of a rural Brazilian community (Cássia dos Coqueiros, SP) determined by Elisa and immunoblotting using Taenia crassiceps antigens. Rev Inst Med Trop São Paulo. 2002; 44: 7-12.

Brucki SM, Nitrini R, Caramelli P, Bertolucci PH, Okamoto IH. Suggestions for utilization of the mini-mental state examination in Brazil. Arq Neuropsiquiatr. 2003; 61: 777-81.

Canelas HM. Neurocisticercose: Incidência, Diagnóstico e Formas Clínicas. Arq Neuropsiquiatr. 1962; 20: 1-16.

Carpio A. Neurocysticercosis: an update. Lancet Infect Dis. 2002; 2: 751-62.

Carpio A, Hauser WA. Prognosis for seizure recurrence in patients with newly diagnosed neurocysticercosis. Neurology. 2002; 59: 1730-4.

Del Brutto OH, Rajshekhar V, White AC Jr, Tsang VC, Nash TE, Takayanagui OM, Schantz PM, Evans CA, Flisser A, Correa D, Botero D, Allan JC, Sarti E, 
Gonzalez AE, Gilman RH, Garcia HH. Proposed diagnostic criteria for neurocysticercosis. Neurology. $2001 \mathrm{Jul} ; 57$ : 177-83.

Del la Garza Y, Graviss EA, Daver NG, Gambarin KJ, Shandera WX, Schantz PM, White AC Jr. Epidemiology of neurocysticercosis in Houston, Texas. Am J Trop Med Hyg. 2005; 73: 766-70.

Ewing JA. Detecting Alcoholism: The CAGE Questionaire. JAMA 1984; 252: 1905- 7.

Folstein MF. "Mini-mental state". A practical method for grading the cognitive state of patients for the clinician. J Psychiatr Res. 1975; 12: 189-98.

Forlenza OV, Filho AH, Nobrega JP, dos Ramos Machado L, de Barros NG, de Camargo CH, da Silva MF. Psychiatric manifestations of neurocysticercosis: a study of 38 patients from a neurology clinic in Brazil. J Neurol Neurosurg Psychiatry. 1997; 62: 612-6.

Forlenza OV, Vieira Filho AH, Machado Ldos R, Nóbrega JP, de Barros NG. [Depressive disorders associated with neurocysticercosis: prevalence and clinical correlations]. Arq Neuropsiquiatr. 1998; 56: 45-52.

Garcia HH, Pretell EJ, Gilman RH, Martinez SM, Moulton LH, Del Brutto OH, Herrera G, Evans CA, Gonzalez AE; Cysticercosis Working Group in Peru. A trial ofantiparasitic treatment to reduce the rate of seizures due to cerebral cysticercosis. N Engl J Med. 2004; 350: 249-5.

Garcia HH, Del Brutto OH; Cysticercosis Working Group in Peru. Neurocysticercosis: updated concepts about an old disease. Lancet Neurol. 2005; 4: 653-61. 
Góngora-Rivera F, Soto-Hernández JL, Guevara P, Sotelo-Morales J. In neurocysticercosis, CSF cytokines correlate with cerebral blood flow velocities. Neurology. 2008; 71: 1119-22.

Griesinger W. Fortgesetzte Beobachtung uber Hirnkrankheiten, Cysticerken und ihre Diagnose. Arch Kinderheilk. 1862; 3: 207-40.

Huang CW, Hsieh YJ, Tsai JJ, Pai MC. Cognitive performance in cryptogenic epilepsy. Acta Neurol Scand. 2005;112: 228-33.

Kraft R. Cysticercosis: An Emerging Parasitic Disease. Am Fam Physician 2007; 76: 91-6.

Lefèvre AB. Distúrbios Psíquicos na Neurocisticercose em Crianças. Arq Neuropsiquiatr. 1969; 27: 103-8.

Lucato LT, Guedes MS, Sato JR, Bacheschi LA, Machado LR, Leite CC. The role of conventional MR imaging sequences in the evaluation of neurocysticercosis: Impact on characterization of the scolex and lesion burden. Am J Neuroradiol. 2007; 28: 1501-4.

Más-Sesé G, Vives-Píera I, Fernandez-Barreiro A, Martinez-Lage JF, Martenez-Salcedo E, Alarcon-Martinez H, Domingo-Jimenez R, Puche-Mira A, Casas-Fernandez C. A descriptive study of neurocysticercosis in a tertiary care hospital. Rev Neurol. 2008; 46: 194-6.

Medina MT, Durín RM, Martínez L, Osorio JR, Estrada AL, Zíniga C, Cartagena D, Collins JS, Holden KR. Prevalence, incidence, and etiology of epilepsies in rural Honduras: the Salama Study. Epilepsia. 2005; 46: 124-31. 
Montano SM, Villaran MV, Ylquimiche L, Figueroa JJ, Rodriguez S, Bautista CT, Gonzalez AE, Tsang VC, Gilman RH, Garcia HH; Cysticercosis Working Group in Peru. Neurocysticercosis: association between seizures, serology and brain CT in rural Peru. Neurology. 2005 Jul 26;65:229-33.

Morris JC. The Consortium to Establish a Registry for Alzheimer's Disease (CERAD) Part 1. Clinical and neuropsychological assessment of Alzheimer's disease. Neurology. 1989; 39: 1159-65.

Nash TE, Del Brutto OH, Butman JA, Corona T, Delgado-Escueta A, Duron RM, Evans CA, Gilman RH, Gonzalez AE, Loeb JA, Medina MT, PietschEscueta S, Pretell EJ, Takayanagui OM, Theodore W, Tsang VC, Garcia HH. Calcific neurocysticercosis and epileptogenesis. Neurology. 2004; 62: 1934-8.

Nitrini R, Lefèvre BH, Mathias SC, Caramelli P, Carrilho PE, Sauaia N, Massad E, Takiguti C, Silva IO, Porto CS, Magila MC, Scaff M. Testes neuropsicológicos de aplicação simples para o diagnóstico de demência. Arq Neuropsiq. 1994; 52: 457-65.

Nitrini R, Caramelli P, Herrera Junior E, et al Performance of illiterate and literate nondemented elderly subjects in two tests of long-term memory. J Int Neuropsychol Soc. 2004; 10: 634-38.

Nitrini R, Caramelli P, Bottino CM, Damasceno BP, Brucki SM, Anghinah R. Academia Brasileira de Neurologia. Diagnosis of Alzheimer's disease in Brazil: cognitive and functional evaluation. Recommendations of the Scientific Department of Cognitive Neurology and Aging of the Brazilian Academy of Neurology. Arq Neuropsiquiatr. 2005; 63: 720-27. 
Pfeffer RI, Kurosaki TT, Harrah CH Jr, Chance JM, Filos S. Measurement of functional activities in older adults in the community. J Gerontol. 1982; 37: 323-29.

Proposal for revised clinical and electroencephalographic classification of epileptic seizures. From the Commission on Classification and Terminology of the International League Against Epilepsy. Epilepsia. 1981; 22: 489-501.

Proposal for classification of epilepsies and epileptic syndromes. Commission on Classification and Terminology of the International League Against Epilepsy. Epilepsia. 1985; 26: 268-78.

Proposal for revised classification of epilepsies and epileptic syndromes. Commission on Classification and Terminology of the International League Against Epilepsy. Epilepsia. 1989; 30: 389-99.

Ramirez-Bermudez J, Higuera J, Sosa AL, Lopez-Meza E, Lopez-Gomez M, Corona T. Is dementia reversible in patients with neurocysticercosis? J Neurol Neurosurg Psychiatry. 2005; 76: 1164-6.

Roman G, Sotelo J, Del Brutto O, Flisser A, Dumas M, Wadia N, Botero D, Cruz M, Garcia H, de Bittencourt PR, Trelles L, Arriagada C, Lorenzana P, Nash TE, Spina-França A. A proposal to declare neurocysticercosis an international reportable disease. Bull World Health Organ. 2000; 78: 399-406.

Saunders JB, Aasland OG, Babor TF, de la Fuente JR, Grant M. Development of the Alcohol Use Disorders Identification Test (AUDIT): WHO collaborative project on early detection of persons with harmful alcohol consumption. II. Addiction. 1993; 88: 791-804. 
Singh S, Dhikav V, Agarwal N, Anand KS. An unusual cause of psychosis. Lancet. 2004; 363: 1522.

Sotelo J, Guerrero V, Rubio F. Neurocysticercosis: a new classification based on active and inactive forms. A study of 753 cases. Arch Intern Med. 1985; 145: 442-45.

Sotelo J. Neurocisticercose: controvérsias. Neuroinfecção. 1996; 1996: 245-52.

Sotelo J. Neurocysticercosis--is the elimination of parasites beneficial? N Engl J Med. 2004; 350: 280-2.

Stroop R. Studies of interference in serial verbal reactions. Journal of Experimentam Psychology. 1935; 18: 643-62.

Takayanagui OM, Jardim E. [Clinical aspects of neurocysticercosis: analysis of 500 cases]. Arq Neuropsiquiatr. 1983; 41: 50-63.

Takayanagui OM, Leite JP. [Neurocysticercosis]. Rev Soc Bras Med Trop.2001; 34: 283-90.

Terra-Bustamante VC, Coimbra ER, Rezek KO, Escorsi-Rosset SR, Guarnieri R, Dalmagro CL, Inuzuka LM, Bianchin MM, Wichert-Ana L, Alexandre V, Takayanagui OM, Araújo D, dos Santos AC, Carlotti CG, Walz R, Markowitsch HJ, Sakamoto AC. Cognitive performance of patients with mesial temporal lobe epilepsy and incidental calcified neurocysticercosis. J Neurol Neurosurg Psychiatry. 2005; 76: 1080-3. 
Tretiakoff C, Pacheco, Silva AC. Contribuição para o estudo da cysticercose cerebral e em particular das lesões cerebrais tóxicas à distância n'esta afecção. Memorias do Hospício de Juqueri 1924; 1: 37-66.

Trzepacz PT, Baker RW, Greenhouse J. A symptom rating scale for delirium. Psychiatry Res 1988; 23: 89-97.

Vaz AJ. Neurocisticercose: Aspectos Biológicos, Imunológicos e Epidemiológicos. Neuroinfecção. 1996; 1996: 181-92.

Wallin M, Kurtzke JF. Neurocysticercosis in the United States: Review of an important emerging infection. Neurology. 2004; 63: 1559-64.

Wechsler D. Wechsler Memory Scale - Revised. San Antonio: Psychological Corporation; 1997.

Zee CS, Go JL, Kim PE, DiGiorgio CM. Imaging of neurocysticercosis. Neuroimaging Clin N Am. 2000; 10: 391-407. 
ANEXO 1

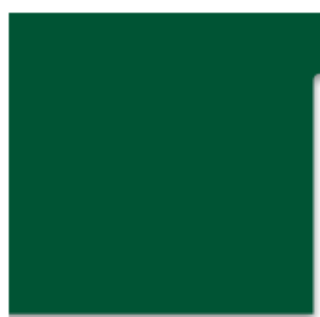

D. Ciampi de Andrade, $\mathrm{MD}^{*}$

C.L. Rodrigues, MD*

R. Abraham, MD, PhD

L.H.M. Castro, MD,

$\mathrm{PhD}$

J.A. Livramento, MD,

$\mathrm{PhD}$

L.R. Machado, MD,

$\mathrm{PhD}$

C.C. Leite, MD, PhD

P. Caramelli, MD, PhD

Address correspondence and ecpriser equess to Dt. Paula Internal Madicins, Faculy of Medicine, Federal Unainersity of Minas Genzi, Av. Alfrodo Bal 190, Room 246, 30.130-900. cormenteresp br

\section{Cognitive impairment and dementia in neurocysticercosis}

A cross-sectional controlled study

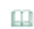

\section{ABSTRACT}

Objectives: Neurocysticercosis (NCYST) is the most frequent CNS parasitic disease worldwide, affecting more than 50 million people. However, some of its clinical findings, such as cognitive impairment and dementia, remain poorly characterized, with no controlled studies conducted so far. We investigated the frequency and the clinical profile of cognitive impairment and dementia in a sample of patients with NCYST in comparison with cognitively healthy controls $(\mathrm{HC})$ and patients with cryptogenic epilepsy (CE).

Methods: Forty treatment-naive patients with NCYST, aged $39.25 \pm 10.50$ years and fulfilling absolute criteria for definitive active NCYST on MRI, were submitted to a comprehensive cognitive and functional evaluation and were compared with $49 \mathrm{HC}$ and 28 patients with $\mathrm{CE}$ of similar age, educational level, and seizure frequency.

Results: Patients with NCYST displayed significant impairment in executive functions, verbal and nonverbal memory, constructive praxis, and verbal fluency when compared with $\mathrm{HC}(\mathrm{p}<0.05)$. Dementia was diagnosed in $12.5 \%$ patients with NCYST according to DSM-IV criteria. When compared with patients with CE, patients with NCYST presented altered working and episodic verbal memory, executive functions, naming, verbal fluency, constructive praxis, and visualspatial orientation. No correlation emerged between cognitive scores and number, localization, or type of NCYST lesions on MRI.

Conclusions: Cognitive impairment was ubiquitous in this sample of patients with active neurocysticercosis (NCYST). Antiepileptic drug use and seizure frequency could not account for these features. Dementia was present in a significant proportion of patients. These data broaden our knowledge on the clinical presentations of NCYST and its impact in world public health.

Neurology 2010:74:1288-1295

\section{GLOSSARY}

AED - antiepileptic drug: BCSB - Brief Cognitive Screening Battery; BNT - Boston Naming Test: CDT - clock-drawing test: CE - cryptogenic Eplepsy: CERAD - Consortium to Establish a Registry for A Azheimer's Disease; CF - category fluency tests; $\mathrm{Cl}$ - canfidenter interval, CIND - cognitive impaiment tho dementia; CP - construetive praxis; DSB - digit-spa backward, DSF = digit-span forward; DSM-N = Diagnastic and Statisticat Manual of Mental Disarders, 4th edition; $\mathbf{H C}$

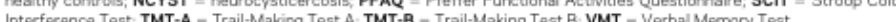

Neurocysticercosis (NCYST) is the most frequent parasitic disease of the CNS, being highly prevalent in Central and South America, Asia, and Africa. ${ }^{1,2}$ However, in recent decades, an increasing number of cases have been reported in the United States ${ }^{3}$ and Europe.

The most frequent clinical presentations of NCYST are symptomatic seizures, epilepsy, focal neurologic deficits, and headache secondary to intracranial hypertension. ${ }^{1.5}$ Diagnosis of NCYST is rarely straightforward, even in patients with epidemiologic risk factors and classic clinical signs in endemic areas. ${ }^{6}$ Significant evidence has been cumulated recently on the patho-

Supplemental data at

Theie authos wertibuted cumally to the suldy.

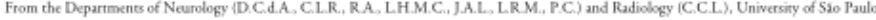

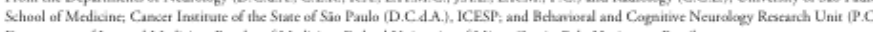
Department of Intemal Mid Srudy finding Suppored by the Department of Neurology of the Univessity of S2o Palsin.

128

Copyright $\odot$ by AAN Enterprises, Inc. Unauthorized reproduction of this article is prohibited. 